\title{
A nearly analytic exponential time difference method for solving 2D seismic wave equations
}

\author{
Xiao Zhang $\cdot$ Dinghui Yang $\cdot$ Guojie Song
}

Received: 24 July 2013/ Accepted: 16 December 2013/Published online: 22 January 2014

(C) The Seismological Society of China, Institute of Geophysics, China Earthquake Administration and Springer-Verlag Berlin Heidelberg 2014

\begin{abstract}
In this paper, we propose a nearly analytic exponential time difference (NETD) method for solving the $2 \mathrm{D}$ acoustic and elastic wave equations. In this method, we use the nearly analytic discrete operator to approximate the high-order spatial differential operators and transform the seismic wave equations into semi-discrete ordinary differential equations (ODEs). Then, the converted ODE system is solved by the exponential time difference (ETD) method. We investigate the properties of NETD in detail, including the stability condition for 1-D and 2-D cases, the theoretical and relative errors, the numerical dispersion relation for the 2-D acoustic case, and the computational efficiency. In order to further validate the method, we apply it to simulating acoustic/elastic wave propagation in multilayer models which have strong contrasts and complex heterogeneous media, e.g., the SEG model and the Marmousi model. From our theoretical analyses and numerical results, the NETD can suppress numerical dispersion effectively by using the displacement and gradient to approximate the high-order spatial derivatives. In addition, because NETD is based on the structure of the Lie group method which preserves the quantitative properties of differential equations, it can achieve more accurate results than the classical methods.
\end{abstract}

Keywords ETD $\cdot$ Lie group method - Numerical approximations and analysis - Computational seismology $\cdot$ Numerical dispersion $\cdot$ Nearly analytic discrete operator

X. Zhang · D. Yang $(\varangle) \cdot$ G. Song

Department of Mathematical Sciences, Tsinghua University, Beijing 100084, China

e-mail: dhyang@math.tsinghua.edu.cn

\section{Introduction}

To determine the Earth's structure, it is necessary to develop an accurate and efficient method for modeling seismic wave propagation in the Earth's media. In this effort, wave field simulation has become a powerful tool in seismological research in the last few decades. To improve the computational accuracy and efficiency of wave field simulation, many numerical techniques have been developed. Currently, widely used methods include the finite difference (FD) method (e.g., Kelly et al. 1976; Dablain 1986; Igel et al. 1995; Blanch and Robertson 1997; Carcione and Helle 1999), the finite element method (e.g., Erikson and Johnson 1991), the pseudo-spectral (PS) method (e.g., Kosloff and Baysal 1982), the spectral element method (e.g., Komatitsch and Vilotte 1998; Komatitsch et al. 2000), the reflectivity method (e.g., Booth and Crampin 1983a, b; Chen 1993), and the boundary integral equation-discrete wavenumber method (e.g., Bouchon 1996; Zhou and Chen 2008). Each method has its own advantages and disadvantages, which we have reviewed briefly in our previous work (Yang et al. 2004).

It is well-known that the FD methods have a number of significant advantages as compared with the other methods due to their easy implement, fast calculations, low computer storage requirements, and high parallelism. Because of these advantages, FD is the method used most frequently in wave field simulations in seismology. However, classical FD methods often produce serious numerical dispersion when computation grids are too coarse or when velocity models have strong contrasts across the interfaces (Dablain 1986; Yang et al. 2006). There are usually two ways to suppress numerical dispersion. One way is to increase the number of spatial sampling grid points in a wavelength, and the other is to use a high-order numerical discrete method. 
Unfortunately, using fine space grids results in a significant increase in memory requirements and computational cost, and a higher-order method does not guarantee lower numerical dispersion (Fei and Larner 1995). To solve the problem, Yang et al. (2003) introduced a type of nearly analytic discrete (NAD) operator to approximate the highorder spatial derivatives. These NAD operators use only three grid points in a spatial direction to achieve the fourthorder spatial accuracy, and operators can efficiently suppress the numerical dispersion. Based on the idea, a range of different effective numerical algorithms have been proposed by Chen et al. (2010), Tong et al. (2011), and Ma et al. (2011).

In the last few years, many numerical integration techniques have been designed to solve the ordinary differential equations (ODEs). These integration methods include the symplectic methods of Hamiltonian systems, the Lie group method (Munthe-Kaas and Zanna 1997; Munthe-Kass 1999; Iserles et al. 2000), the exponential time difference method (Cox and Matthews 2002; Minchev 2003; Krogstad 2005; Sun et al. 2008), and the precise integration method (Tang and Yang 2007; Li et al. 2010). Geometric integration methods usually preserve the important qualitative properties for some special ODEs and have better stability for some differential equations with a special structure. The numerical result of the integration methods is usually more accurate than those of the classical methods.

In this paper, we develop a nearly analytic exponential time difference (NETD) method by combining the basic idea of the Lie group method with the NAD method to solve seismic wave equations, which can effectively suppress the numerical dispersion and preserve the qualitative properties of PDEs. In this method, first, we use the nearly analytic operator to approximate the high-order spatial differential operator and convert the wave equations into semi-discrete ODEs. Then we solve the resulting ODE system using the exponential time difference method. We compare the results from the NETD with those of the Lax-Wendroff correction (LWC) scheme and the staggered grid (SG) scheme. The numerical results computed by NETD are also compared with the exact solution. Our numerical experiments show that the NETD method has weak numerical dispersion both when coarse grids are used and when the velocity models have large contrasts between the neighboring layers. From our process to obtain the NETD method, we can see that the new method is based on the geometric integration methods such as the Lie group method and the ETD method. Since these geometric integration methods can preserve qualitative properties of differential equations, we hope that the NETD method can achieve a more accurate result. As a result, the NETD method has quite a few advantages over other methods such as numerical dispersion, numerical error, and computational cost.

\section{NETD method for the acoustic wave equation}

In this section, we introduce and describe in detail the derivation of the NETD method for solving acoustic wave equation. The method comprises three major steps: the exponential time difference method, the transformation of the wave equations, and the semi-implicit scheme.

\subsection{Exponential time difference method}

Consider the stiff system

$\frac{\mathrm{d} u}{\mathrm{~d} t}=L u+N(u, t)$,

where $L$ is a higher-order linear term and $N$ is a lower-order nonlinear term.

In order to solve Eq. (1), we first multiply the equation by an integrating factor

$\frac{\mathrm{d}}{\mathrm{d} t}(\exp (-L t) u)=\exp (-L t) N(u, t)$

and then integrate Eq. (2) over a single temporal step of length $\tau$,

$$
\begin{aligned}
u^{n+1}= & \exp (L \tau) u^{n}+\exp (L \tau) \\
& \int_{0}^{\tau} \exp (-L s) N\left(u\left(t_{n}+s\right), t_{n}+s\right) \mathrm{d} s .
\end{aligned}
$$

Using the truncated Taylor series expansion of $N(u, t)$ at $t_{n}$ to approximately calculate the integration in Eq. (3), we can obtain a numerical solution of Eq. (1). For example, we let $N\left(u\left(t_{n}+s\right), t_{n}+s\right) \approx N\left(u^{n}, t_{n}\right)$, thus the numerical scheme can be written as

$u^{n+1}=\exp (L \tau) u^{n}+L^{-1}(\exp (L \tau)-I) N\left(u^{n}, t_{n}\right)$.

On the other hand, Scheme (4) can be also obtained from the idea of the Lie group method. We let $G=G L(d) \ltimes R^{d}$ be the semi-direct product of the general linear group and $R^{d}$. The Lie algebra of $G$ is given as $g=g l(d) \ltimes R^{d}$ with the Lie bracket

$[(A, a),(\tilde{A}, \tilde{a})]=([A, \tilde{A}], A \tilde{a}-\tilde{A} a)$,

and exponential mapping

$\exp (A, a)=\left(\exp (A), A^{-1}(\exp (A)-I) a\right)$.

Now we can rewrite Eq. (1) in the form

$\frac{\mathrm{d} u}{\mathrm{~d} t}=(L, N(u, t)) \cdot u=F_{u, t}(u)$,

where $(A, a) \cdot u=A u+a$ and where the algebra action is given as 


$$
\begin{aligned}
\lambda((A, a), u) & =\Lambda(\exp (A, a), u) \\
& =\exp (A) u+A^{-1}(\exp (A)-I) a .
\end{aligned}
$$

Now Eq. (7) can be solved under the Lie group structure. To solve the equation, the "frozen vector field" technique is used here. Speaking in detail, let $F_{\hat{u}, \hat{t}}(u)$ be the frozen vector field at the point $(\hat{u}, \hat{t})$,

$F_{\hat{u}, \hat{t}}(\hat{u})=(L, N(\hat{u}, \hat{t})) \cdot u=L u+N(\hat{u}, \hat{t})$.

The flow of this vector field is

$$
\begin{aligned}
u^{n+1} & =\lambda\left(\tau F_{\hat{u}, \hat{t}}, u^{n}\right)=\Lambda\left(\exp \left(\tau F_{\hat{u}, \hat{t}}\right), u^{n}\right) \\
& =\exp (L \tau) u^{n}+L^{-1}(\exp (L \tau)-I) N(\hat{u}, \hat{t}) .
\end{aligned}
$$

Let $\hat{u}=u^{n}, \hat{t}=t_{n}$, thus Eq. (10) can be converted to Scheme (4).

\subsection{Transformation of wave equations}

The acoustic wave equation in a 2-D homogeneous isotropic medium is written as

$\frac{\partial^{2} u}{\partial t^{2}}=c_{0}^{2} \Delta u$

where $u$ and $c_{0}$ are the displacement and the acoustic velocity, respectively, and $\Delta=\frac{\partial^{2}}{\partial x^{2}}+\frac{\partial^{2}}{\partial z^{2}}$ is the Laplace operator.

Let

$w=\frac{\partial u}{\partial t}, \quad U=\left(u, \frac{\partial u}{\partial x}, \frac{\partial u}{\partial z}\right)^{\mathrm{T}}, \quad W=\left(w, \frac{\partial w}{\partial x}, \frac{\partial w}{\partial z}\right)^{\mathrm{T}}$.

Equation (11) can be rewritten as

$$
\left\{\begin{array}{l}
\frac{\partial U}{\partial t}=W \\
\frac{\partial W}{\partial t}=c_{0}^{2}\left(\frac{\partial^{2} U}{\partial x^{2}}+\frac{\partial^{2} U}{\partial z^{2}}\right)
\end{array}\right.
$$

We use the fourth-order NAD operator (see Appendix 1 for details) to approximate the high-order spatial derivatives in Eq. (12). Then Eq. (12) can be transformed into an ODE system as follows:

$\frac{\mathrm{d}}{\mathrm{d} t}\left(\begin{array}{c}U \\ W\end{array}\right)_{j, k}=\left(\begin{array}{cc}0 & \boldsymbol{I} \\ \boldsymbol{\Omega} & 0\end{array}\right)\left(\begin{array}{c}U \\ W\end{array}\right)_{j, k}+N(U, W)$.

The first part of the right side of Eq. (13) is a linear combination of $u, w$ and their gradient at the grid point $\left(x_{j}, z_{k}\right)$, and the second part is a linear combination of $u, w$ at its neighboring grid point. $I$ is a $3 \times 3$ identity matrix and $\Omega$ is a $3 \times 3$ matrix that depends on the spatial discrete operator.
We let

$Y_{j, k}=(U, W)_{j, k}^{T}, \quad L=\left(\begin{array}{cc}0 & I \\ \Omega & 0\end{array}\right)$

and regard $N$ as a nonlinear function of $Y_{j, k}$. Therefore, Eq. (13) can be rewritten as

$\frac{\mathrm{d} Y_{j, k}}{\mathrm{~d} t}=L Y_{j, k}+N\left(Y_{j, k}\right)$.

Now Eq. (14) can be solved by the exponential time difference method. For instance, we can write a numerical scheme

$Y_{j, k}^{n+1}=\exp (L \Delta t) Y_{j, k}^{n}+L^{-1}(\exp (L \Delta t)-I) N\left(Y^{n}\right)$,

where $\Delta t$ is the temporal step size.

\subsection{Implicit scheme}

In order to improve the stability of the NETD method, we reform Scheme (15) into an implicit scheme. By replacing $Y^{n}$ with $\frac{1}{2}\left(Y^{n+1}+Y^{n}\right)$, we can rewrite Scheme (15) as

$$
\begin{aligned}
& \left(I-\frac{1}{2} L^{-1}(\exp (L \Delta t)-I) N\right) Y^{n+1} \\
& =\left(\exp (L \Delta t)+\frac{1}{2} L^{-1}(\exp (L \Delta t)-I) N\right) Y^{n} .
\end{aligned}
$$

Let $Y^{*}=\left(I-\frac{1}{2} L^{-1}(\exp (L \Delta t)-I) N\right) Y^{n+1}$, such that Eq. (16) can be rewritten as follows:

$$
\left\{\begin{aligned}
Y^{*}= & \left(\exp (L \Delta t)+\frac{1}{2} L^{-1}(\exp (L \Delta t)-I) N\right) Y^{n} \\
& \left(I-\frac{1}{2} L^{-1}(\exp (L \Delta t)-I) N\right) Y^{n+1}=Y^{*} .
\end{aligned}\right.
$$

Now we can explicitly solve the system of linear equations in Scheme (17). Let

$\frac{1}{2} L^{-1}(\exp (L \Delta t)-I) N=B$,

and use the truncated power series expansion

$(I-B)^{-1} \approx I+B+B^{2}$.

Thus, we can rewrite Scheme (17) explicitly as

$$
\left\{\begin{array}{l}
Y^{*}=\left(\exp (L \Delta t)+\frac{1}{2} L^{-1}(\exp (L \Delta t)-I) N\right) Y^{n} \\
Y^{n+1}=\left(I+B+B^{2}\right) Y^{*} .
\end{array}\right.
$$

\section{NETD method for elastic wave equations}

In this section, we briefly introduce the NETD method for solving elastic wave equations. In a 2-D anisotropic medium, the elastic wave equation can be written as 
$\frac{\partial \sigma_{i j}}{\partial x_{j}}+f_{i}=\rho \frac{\partial^{2} u_{i}}{\partial t^{2}}$,

where $\rho(x, z)$ is the density, $u_{i}$ and $f_{i}$ are the displacement and the force component in the $i$ th direction, respectively, and $\sigma_{i j}$ is the stress tensor.

Using the stress-strain relation, we can transform Eq. (19) into the following vector equation:

$\rho \frac{\partial^{2} U}{\partial t^{2}}=D U+f$,

where $U=\left(u_{1}, u_{2}, u_{3}\right)^{\mathrm{T}}, f=\left(f_{1}, f_{2}, f_{3}\right)^{\mathrm{T}}$, and $D$ is the second-order spatial partial differential operator.

Let $W=\frac{\partial U}{\partial t}$, then Eq. (20) can be rewritten as

$\left\{\begin{array}{l}\frac{\partial U}{\partial t}=W \\ \frac{\partial W}{\partial t}=\frac{1}{\rho} D U+\frac{1}{\rho} f .\end{array}\right.$.

Equation (21) can be solved by the NETD method as the previous.

\section{Stability criteria}

It is important to keep the numerical calculation stable; therefore, it is necessary to investigate the stability condition of the NETD method. Following the analysis process presented by Yang et al. (2006), we perform a series of mathematical derivations, and thereby obtain the stability condition of the NETD method for solving acoustic wave equations in 1-D and 2-D acoustic cases.

For 1-D homogeneous cases, the Courant number is restricted by

$\alpha=c_{0} \Delta t / h \leq 0.4232$.

We assume that the computational domain is subdivided by uniform grids $(h=\Delta x=\Delta z)$, such that the stability condition of the NETD method for solving 2-D acoustic wave equations in homogenous media is given by

$\alpha=c_{0} \Delta t / h \leq 0.2583$.

Note that the maximal Courant number of NETD for the 2-D case is relatively compact, which means the time step maybe restricted to relatively small values in some cases. As a result, the iterative steps in time advancing may be increased.

It is usually difficult to determine the stability condition for heterogeneous cases. However, it can be approximated using a local homogeneous case. We assume that Eqs. (22) and (23) are approximately correct for a heterogeneous medium if the maximal wave velocity $c_{0}$ is used.

\section{Error analysis}

\subsection{Theoretical analysis}

In this section, we first analyze the theoretical error of the NETD method. Using the Taylor series expansion, we conclude that the NETD method is fourth-order accurate with respect to space. From the truncated Taylor series expansion of $N(u, t)$ suggested by Krogstad (2005), we can know that the truncation error of Scheme (3) is $O\left(\Delta t^{2}\right)$.

\subsection{Numerical errors}

To further investigate the numerical errors of the NETD method, we consider the following initial 2-D problem:

$$
\left\{\begin{array}{l}
\frac{\partial^{2} u}{\partial x^{2}}+\frac{\partial^{2} u}{\partial z^{2}}=\frac{1}{c_{0}^{2}} \frac{\partial^{2} u}{\partial t^{2}} \\
u(x, z, 0)=\cos \left(-\frac{2 \pi f_{0}}{c_{0}} x \cos \theta_{0}-\frac{2 \pi f_{0}}{c_{0}} z \sin \theta_{0}\right) \\
\frac{\partial u(x, z, 0)}{\partial t}=-2 \pi f_{0} \sin \left(-\frac{2 \pi f_{0}}{c_{0}} x \cos \theta_{0}-\frac{2 \pi f_{0}}{c_{0}} z \sin \theta_{0}\right)
\end{array},\right.
$$

where $c_{0}$ is the velocity of the plane wave, $\theta_{0}$ is the incident angle at time $t=0$, and $f_{0}$ is the frequency. The exact solution of this initial problem is

$u(x, z, t)=\cos \left[2 \pi f_{0}\left(t-\frac{x}{c_{0}} \cos \theta_{0}-\frac{z}{c_{0}} \sin \theta_{0}\right)\right]$.

In the numerical experiment, we choose the computational domain as $0<x \leq 6 \mathrm{~km}, 0<z \leq 6 \mathrm{~km}$, the frequency is $f_{0}=30 \mathrm{~Hz}$, the wave velocity $c_{0}=4,000 \mathrm{~m} / \mathrm{s}$, and the angle $\theta_{0}=\pi / 4$. The relative error $\left(E_{\mathrm{r}}\right)$ for the 2-D case is defined by

$$
\begin{aligned}
E_{\mathrm{r}}(\%)= & \left\{\frac{1}{\sum_{i=1}^{N} \sum_{j=1}^{N}\left[u\left(x_{i}, z_{j}, t_{n}\right)\right]^{2}}\right. \\
& \left.\sum_{i=1}^{N} \sum_{j=1}^{N}\left[u_{i, j}^{n}-u\left(x_{i}, z_{j}, t_{n}\right)\right]^{2}\right\}^{\frac{1}{2}} .
\end{aligned}
$$

Figure 1 shows the computational results of the relative error $E_{\mathrm{r}}$ at different times under the condition of $\Delta x=\Delta z$. The three lines of $E_{\mathrm{r}}$ corresponding to the SG method with the fourth-order accuracy, the LWC method with the fourth-order accuracy, and the NETD method, respectively, are shown in a semi-log scale in Fig. 1. In Fig. 1a, we choose a spatial grid size of $\Delta x=$ $\Delta z=40 \mathrm{~m}$ and the number of grid points as $151 \times 151$. The spatial size, as shown in Fig. 1b, is $\Delta x=\Delta z=30 \mathrm{~m}$, and the number of grid points is $201 \times 201$. In the calculations, we fix the same time grid size of $\Delta t=5 \times 10^{-4} \mathrm{~s}$ for both the 
numerical experiments. From the figures, it is evident that of the three methods the NETD method has the smallest relative error $E_{\mathrm{r}}$ for the chosen computational parameters. Thus, it is

Table 1 Numerical errors and convergence orders of the NETD method

\begin{tabular}{lllll}
\hline$h$ & $E_{L^{1}}$ & $E_{L^{2}}$ & $O_{L^{1}}$ & $O_{L^{2}}$ \\
\hline $2.000 \mathrm{E}-01$ & $2.379 \mathrm{E}-01$ & $9.053 \mathrm{E}-02$ & & \\
$1.000 \mathrm{E}-01$ & $1.162 \mathrm{E}-02$ & $6.201 \mathrm{E}-03$ & 4.356 & 3.868 \\
$5.000 \mathrm{E}-02$ & $8.981 \mathrm{E}-04$ & $3.382 \mathrm{E}-04$ & 3.694 & 4.197 \\
$3.333 \mathrm{E}-02$ & $1.596 \mathrm{E}-04$ & $5.983 \mathrm{E}-05$ & 4.158 & 4.169 \\
\hline
\end{tabular}

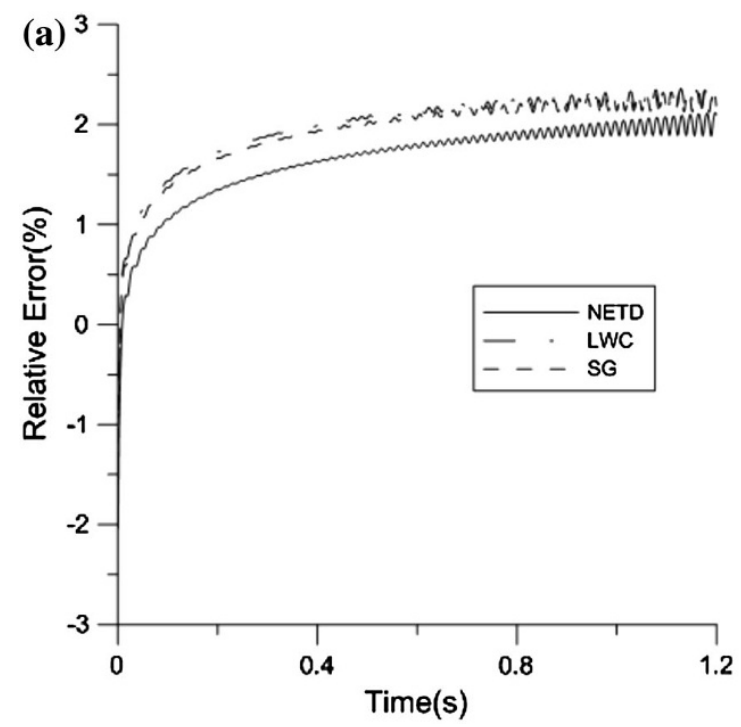

numerically evident that the NETD method is fourth-order accurate with respect to space.

Next, we discuss the order of convergence for the NETD method. We consider the 1-D initial problem:

$$
\left\{\begin{array}{l}
\frac{\partial^{2} u}{\partial x^{2}}=\frac{1}{c_{0}^{2}} \frac{\partial^{2} u}{\partial t^{2}} \\
u(x, 0)=\cos \left(-\frac{2 \pi f_{0}}{c_{0}} x\right) \\
\frac{\partial u(x, 0)}{\partial t}=-2 \pi f_{0} \sin \left(-\frac{2 \pi f_{0}}{c_{0}} x\right) .
\end{array}\right.
$$

The exact solution of the initial problem is

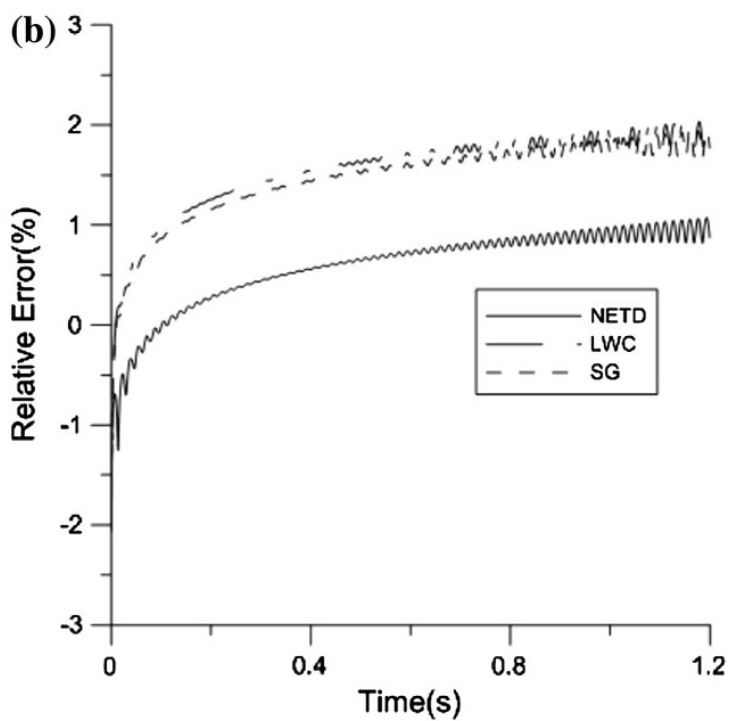

Fig. 1 The relative errors of the fourth-order LWC method, the fourth-order SG method, and the NETD method measured by $E_{\mathrm{r}}$ (Formula (26)) are shown in a semi-log scale for the 2-D initial problem (24). The spatial increments are a $\Delta x=\Delta z=40 \mathrm{~m}$ and $\mathbf{b} \Delta x=\Delta z=30 \mathrm{~m}$, respectively. The time step is $0.5 \mathrm{~ms}$ for both the figures
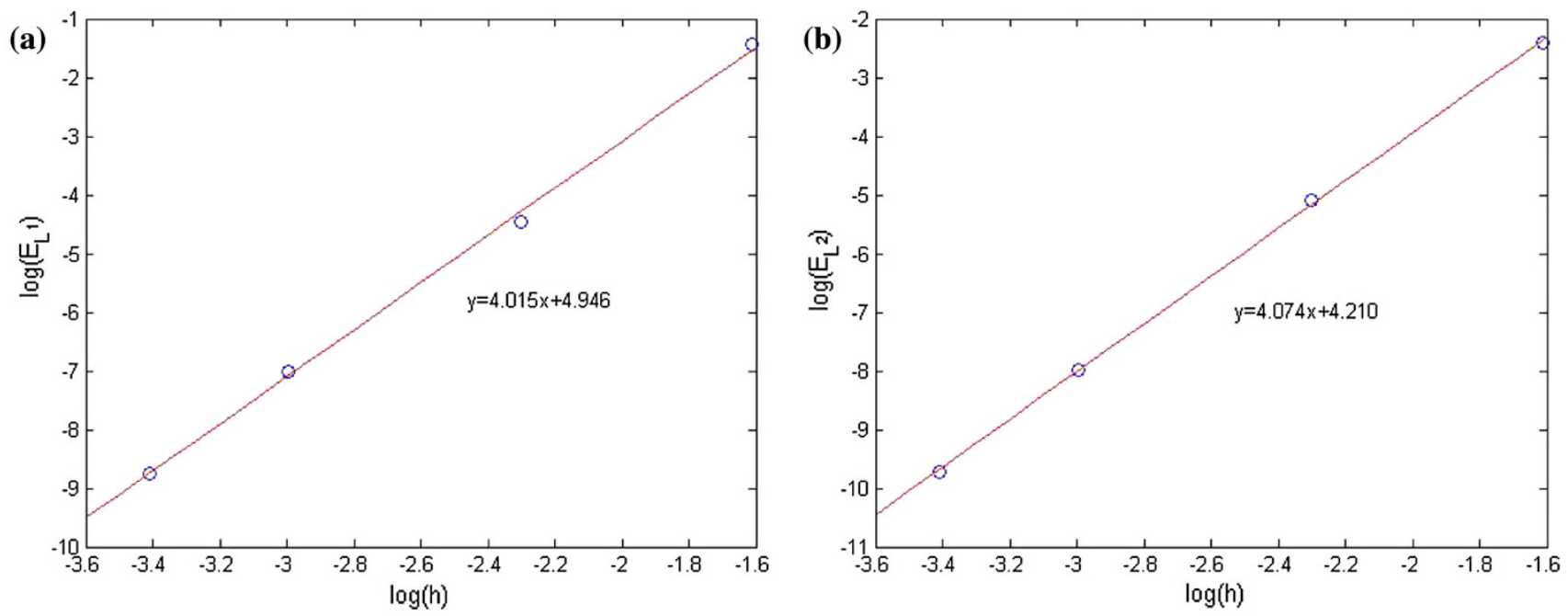

Fig. 2 The $E_{L^{1}}-h$ graph (a) and $E_{L^{2}}-h$ graph (b) in the log-log scale 
$u(x, t)=\cos \left[2 \pi f_{0}\left(t-\frac{x}{c_{0}}\right)\right]$.

We choose the 1-D computational domain as $0<x \leq 10 \mathrm{~km}$ and fix the propagation time at $T=0.5 \mathrm{~s}$.

In this example, we choose a frequency of $f_{0}=4 \mathrm{~Hz}$, and a wave velocity of $c_{0}=4,000 \mathrm{~m} / \mathrm{s}$. In Table 1 , we show the numerical errors of the variable $u$. For the fixed spatial grid size $h$, the error of the numerical solution $u_{h}$ with respect to the exact solution $u$ measured in the discrete $L^{1}, L^{2}$ norms is given by

$E_{L^{k}}=\left(h \sum_{j=1}^{N}\left|u_{h}\left(x_{j}, T\right)-u\left(x_{j}, T\right)\right|^{k}\right)^{\frac{1}{k}}, \quad k=1,2$.

From Table 1, if we choose two different spatial increments $h^{i}, h^{i-1}$ and two errors $E_{L^{k}}^{i}$ and $E_{L^{k}}^{i-1}$ corresponding to $L^{k}$, the orders of numerical convergence can be given as in Dumbser et al. (2007):

$O_{L^{k}}=\log \left(\frac{E_{L^{k}}^{i}}{E_{L^{k}}^{i-1}}\right) / \log \left(\frac{h^{i}}{h^{i-1}}\right), \quad k=1,2$.

Table 1 shows the numerical errors and the convergence orders. The first column shows the spatial increment $h$, and the other four columns show $L^{1}$ and $L^{2}$ errors and the corresponding convergence orders $O_{L^{1}}$ and $O_{L^{2}}$. Figure 2a is the $E_{L^{1}}-h$ graph
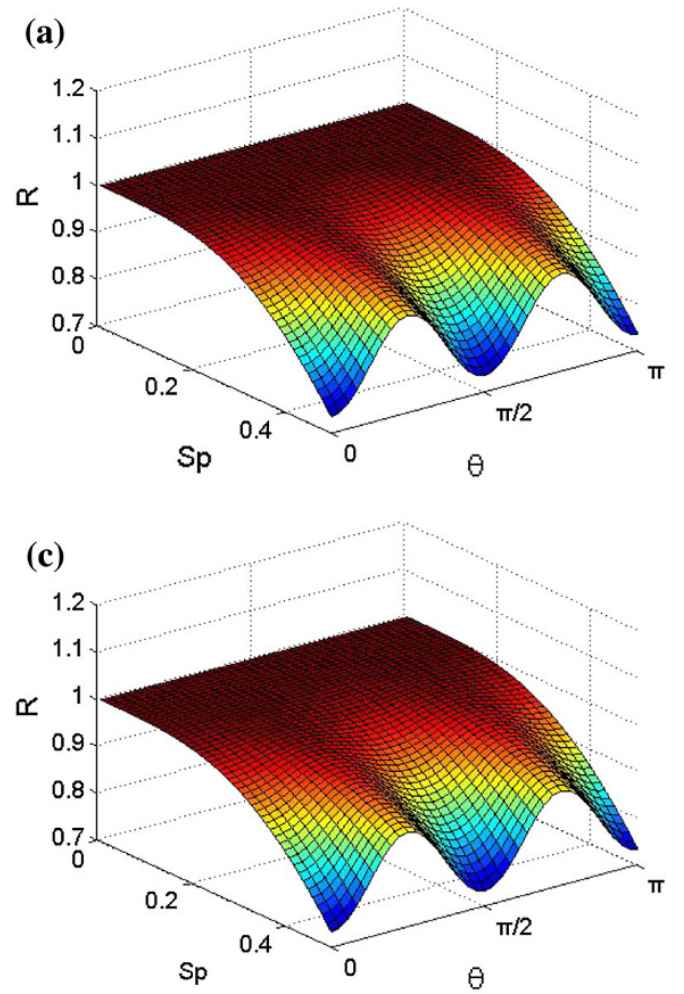

in a $\log -\log$ scale, whereas Fig. $2 \mathrm{~b}$ is the $E_{L^{2}}-h$ graph in a log$\log$ scale. The errors $E_{L^{1}}$ and $E_{L^{2}}$ decrease as the spatial grid size $h$ decreases, and the graphs in Fig. 2 show two straight lines each of which have an approximate slope of 4 . Thus, we can infer that the NETD method is convergent.

\section{Numerical dispersion analysis}

Following the dispersion analysis presented by Dablain (1986) and Yang et al. (2006), we obtain the dispersion relations of the NETD method for the 2-D acoustic wave equations (presented in detail in Appendix 3). We define the spatial sampling ratio first proposed by Moszo et al. (2000) as $S_{\mathrm{p}}=h / \lambda$, in which $\lambda$ denotes the wavelength. The dispersion ratio $R$ is defined as $R=c_{\text {num }} / c_{0}$, , in which $c_{\text {num }}$ and $c_{0}$ denote the numerical and true velocity, respectively. Figures 3, 4, and 5 show the numerical dispersion surfaces for different Courant numbers of LWC, SG, and NETD. In each method, the dispersion curves of a fixed wave propagation direction gradually deviate from $R=1$ as $S_{\mathrm{p}}$ increases. This means the number of grid points in a wavelength decreasing results in a worse approximation. However, we find that the deviation of the entire dispersion surface for NETD is less than that of
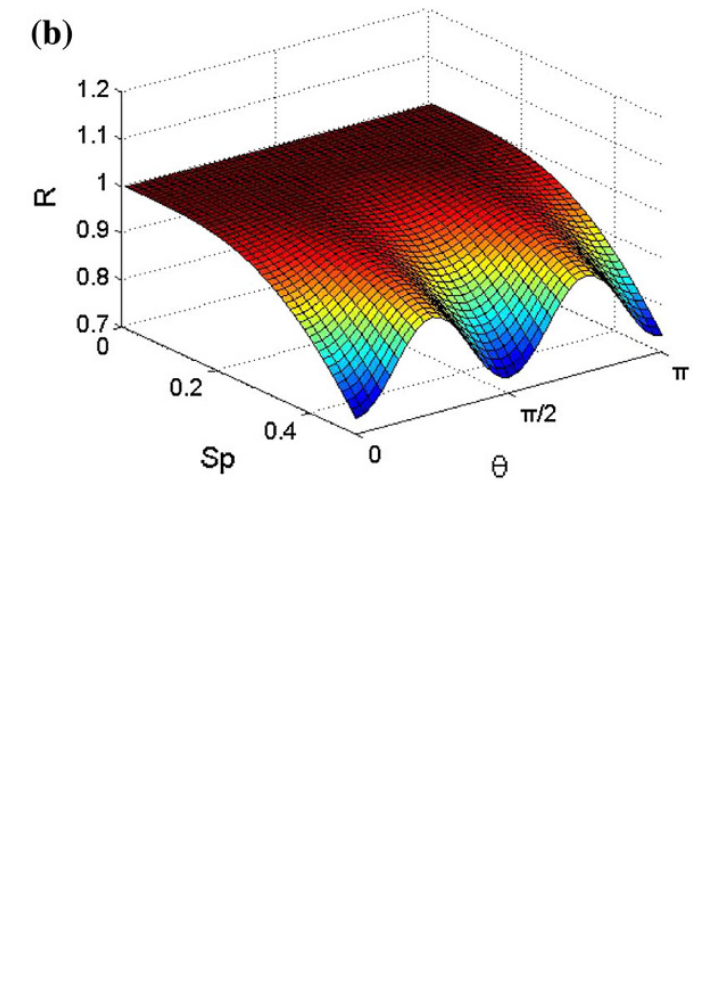

Fig. 3 The ratio $R$ of the numerical wave velocity to the phase velocity versus the sampling rate $S_{\mathrm{p}}$ for different propagation directions for the LWC method. The Courant numbers are $\mathbf{a} \alpha=0.1, \mathbf{b} \alpha=0.15$, and $\mathbf{c} \alpha=0.2$, respectively 

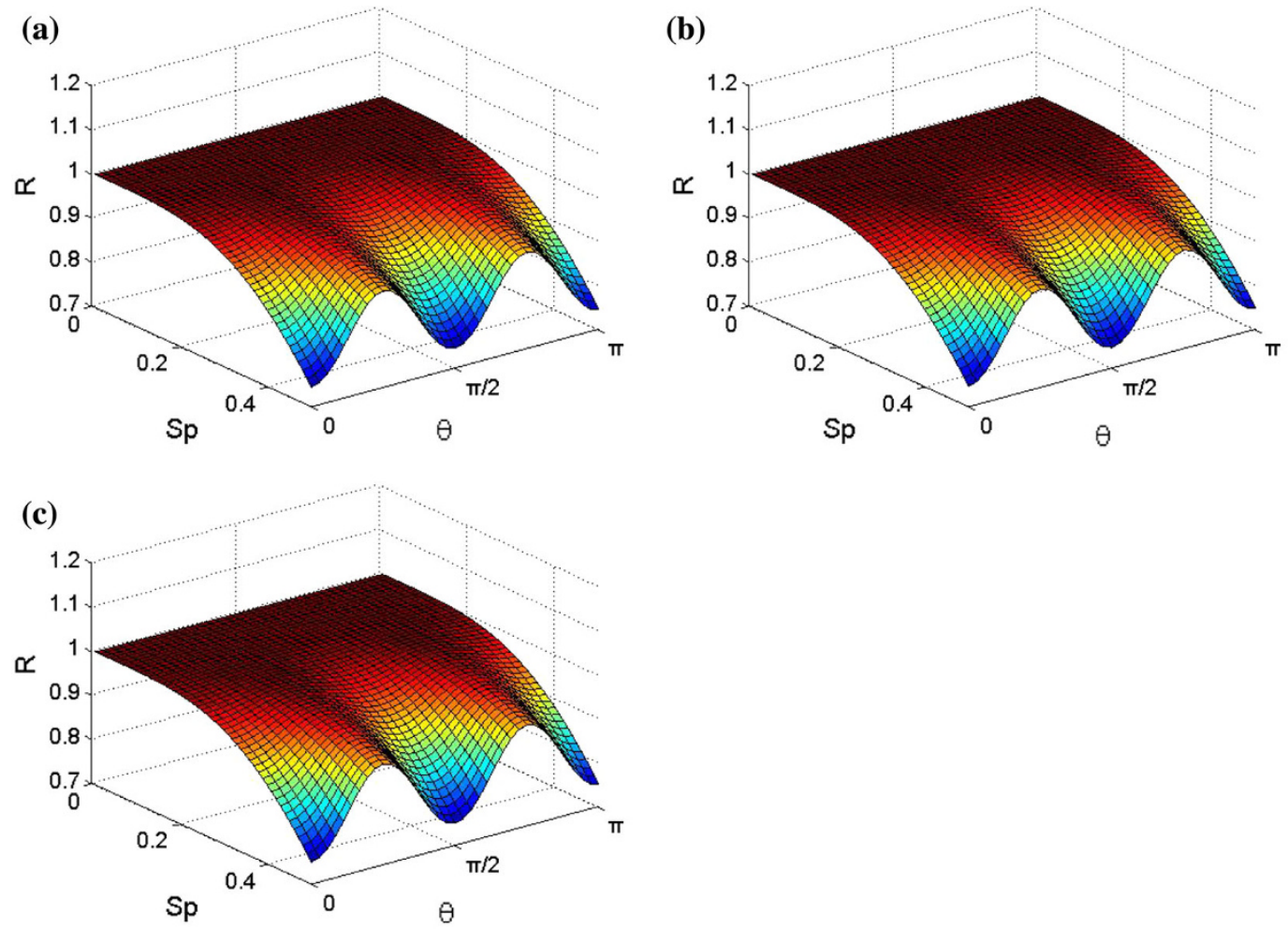

Fig. 4 The ratio $R$ of the numerical wave velocity to the phase velocity versus the sampling rate $S_{\mathrm{p}}$ for different propagation directions for the SG method. The Courant numbers are $\mathbf{a} \alpha=0.1, \mathbf{b} \alpha=0.15$, and $\mathbf{c} \alpha=0.2$, respectively
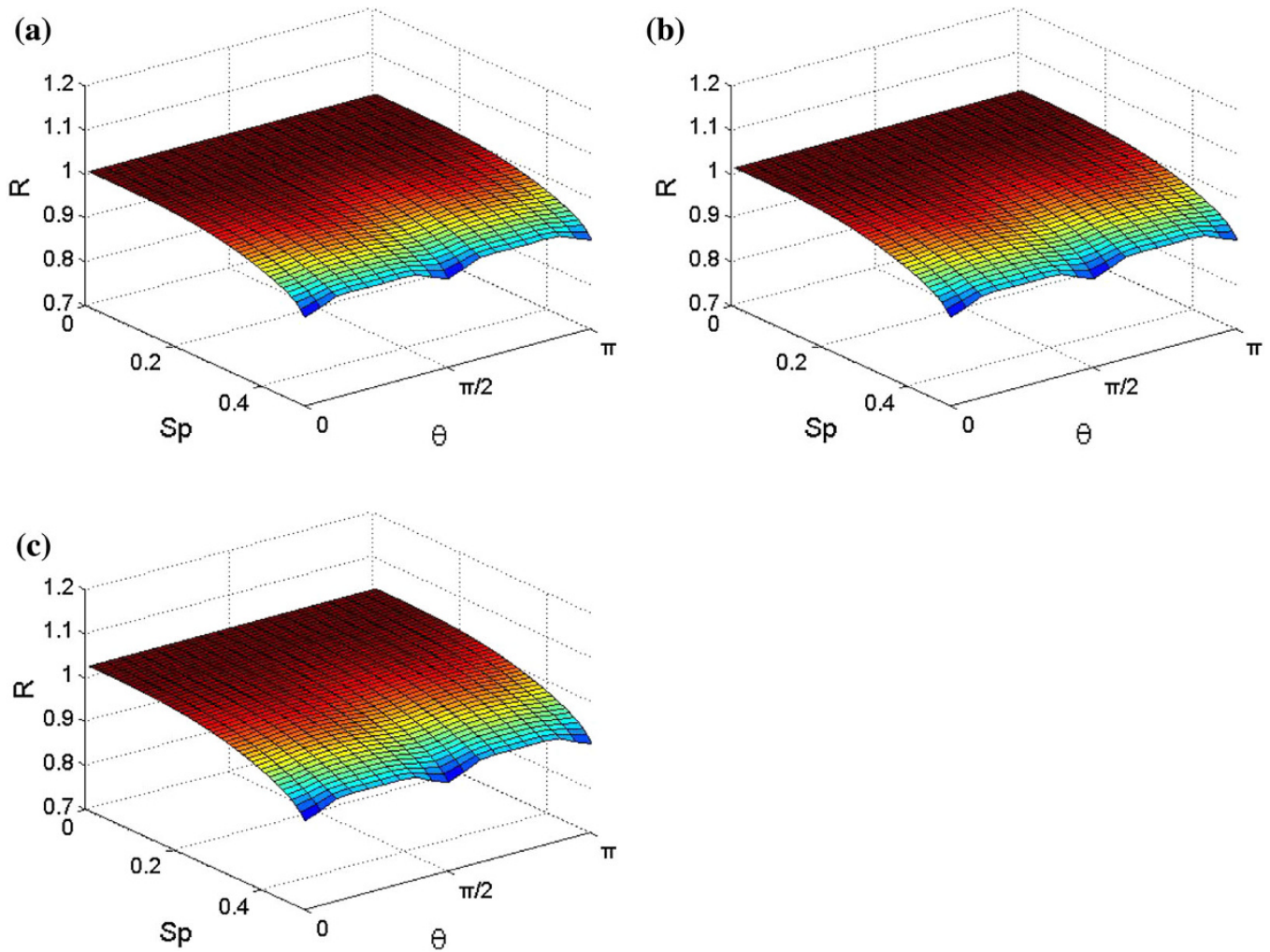

Fig. 5 The ratio $R$ of the numerical wave velocity to the phase velocity versus the sampling rate $S_{\mathrm{p}}$ for different propagation directions for the NETD method. The Courant numbers are $\mathbf{a} \alpha=0.1, \mathbf{b} \alpha=0.15$, and $\mathbf{c} \alpha=0.2$, respectively 
Table 2 The minimum and maximum ratios of the numerical phase velocity to the exact phase velocity for LWC, SG, and NETD for the Courant number $\alpha=0.1,0.15,0.2$

\begin{tabular}{cllll}
\hline \multirow{5}{*}{ Method } & $\alpha$ & & \\
\cline { 3 - 5 } & & 0.10 & 0.15 & 0.20 \\
\hline$R_{\max } / \theta$ & LWC & $1.0000(0,2 \pi)$ & $1.0000(0,2 \pi)$ & $1.0000(0,2 \pi)$ \\
& SG & $1.0000(0,2 \pi)$ & $1.0000(0,2 \pi)$ & $1.0000(0,2 \pi)$ \\
& NETD & $1.0067(0,2 \pi)$ & $1.0153(0,2 \pi)$ & $1.0275(0,2 \pi)$ \\
$R_{\min } / \theta$ & LWC & $0.7342, \pi / 2$ & $0.7332, \pi / 2$ & $0.7316, \pi / 2$ \\
& SG & $0.7444, \pi / 2$ & $0.7466, \pi / 2$ & $0.7496, \pi / 2$ \\
& NETD & $0.9003, \pi / 2$ & $0.9003, \pi / 2$ & $0.9003, \pi / 2$ \\
Error & LWC & 26.57 & 26.67 & 26.83 \\
$(\%)$ & SG & 25.56 & 25.34 & 25.04 \\
& NETD & 9.97 & 9.97 & 9.97
\end{tabular}

The maximum relative error is given by Error $=|R-1|_{\max } \times 100 \%$

LWC and SG. This indicates that of the three methods NETD is the most accurate. In addition, the numerical dispersion for NETD, as compared with those for LWC and SG, has less numerical dispersion anisotropy because its dispersion curve for a fixed sampling rate $S_{\mathrm{p}}$ varies much more slowly with the wave propagation angle $\theta$. Table 2 shows the maximum and minimum dispersion ratios $R_{\max }$ and $R_{\min }$ and the maximum dispersion errors of LWC, SG, and NETD for three Courant numbers as shown in Figs. 3, 4, and 5. From Table 2, it is evident that the dispersion error of NETD is less than $10 \%$ in all directions. However, the maximum relative errors of LWC and SG can be as high as about $25 \%$ in some wave propagation directions.

\section{Comparison with analytic solutions}

To investigate the validity of NETD, in the following section, we compare the numerical solutions generated by NETD with the results from analytic methods, the fourthorder LWC, and the fourth-order SG in a homogeneous medium model and in a two-layer model.

\subsection{Homogeneous medium model}

In this experiment, we compare the numerical solutions computed by NETD with an analytic solution for the acoustic wave equation in a homogeneous medium. Consider Eq. (11) with an explosive source, in this case is a Ricker wavelet with a frequency of $f_{0}=20 \mathrm{~Hz}$. The source function is given by $f(t)=-5.76 f_{0}^{2}\left[1-16\left(0.6 f_{0} t-1\right)^{2}\right] \exp \left[-8\left(0.6 f_{0} t-1\right)^{2}\right]$.

We choose the domain of $0 \leq x \leq 10 \mathrm{~km}$ and $0 \leq z \leq 10 \mathrm{~km}$ with a velocity of $c_{0}=4 \mathrm{~km} / \mathrm{s}$. The source is located at the center of the computational domain, and the receiver is located at $(x=6 \mathrm{~km}, \mathrm{z}=5 \mathrm{~km})$. Figure $6 \mathrm{a}-\mathrm{c}$ shows the waveforms generated by NETD for various spatial and temporal grid sizes and the analytic results at the receiver. In Fig. 6, the spatial steps are chosen as (a) $h=\Delta x=\Delta z=40 \mathrm{~m}$, (b) $h=\Delta x$ $=\Delta z=40 \mathrm{~m}$, and (c) $h=\Delta x=\Delta z=20 \mathrm{~m}$, respectively. The temporal grid sizes are (a) $\Delta t=0.001 \mathrm{~s}$, (b) $\Delta t=5 \times 10^{-4} \mathrm{~s}$, and (c) $\Delta t=2.5 \times 10^{-4} \mathrm{~s}$, respectively. In Fig. $6 \mathrm{a}-\mathrm{c}$, the numerical results computed by the NETD method are gradually identical to those generated by the analytic method as the spatial and temporal grid sizes decrease, indicating both the convergence and the validity of the NETD method.

\subsection{Two-layer model}

In the second example, we compare the numerical solutions computed using the NETD, the fourth-order LWC, and the fourth-order SG with the analytic solution for the acoustic wave equation in a two-layer model. We choose the computational domain of $0 \leq x \leq 20 \mathrm{~km}$ and $0 \leq z \leq 20 \mathrm{~km}$. The velocity for the two-layer model is $2.4 \mathrm{~km} / \mathrm{s}$ in the upper layer and $4.8 \mathrm{~km} / \mathrm{s}$ in the lower layer. The interface is located at a depth of $8 \mathrm{~km}$. The explosive source is a Ricker wavelet with a frequency of $15 \mathrm{~Hz}$. The source is located at $(x=10 \mathrm{~km}, \mathrm{z}=7 \mathrm{~km})$ and the receiver is located at $(\mathrm{x}=10 \mathrm{~km}, \mathrm{z}=6 \mathrm{~km})$. We choose the spatial increment as $\Delta x=\Delta z=40 \mathrm{~m}$ and the temporal increment as $\Delta t=0.001 \mathrm{~s}$, which results in four sampling points in a minimal wavelength. In this experiment, the analytical solution is computed by the Cagniard-de Hoop method (Aki and Richards 1980; Berg et al. 1993).

Figure $7 \mathrm{a}-\mathrm{c}$ presents the comparisons of the waveforms generated by the NETD, the fourth-order LWC, and the fourth-order SG methods; and the analytic solution at the receiver $\mathrm{R}$. The solid lines represent the analytical solution and the dashed lines the numerical solutions, respectively. For the relatively coarse grid (four sampling points per minimal wavelength), the fourth-order LWC and the fourth-order SG suffer from serious numerical dispersions (Fig. 7b, c). Compared with the waveforms of those other methods, the results from NETD and from the analytic method (Fig. 7a) match well. This demonstrates that NETD can provide accurate numerical solutions when coarse grids are used and when there are large velocity contrasts across the interface. 

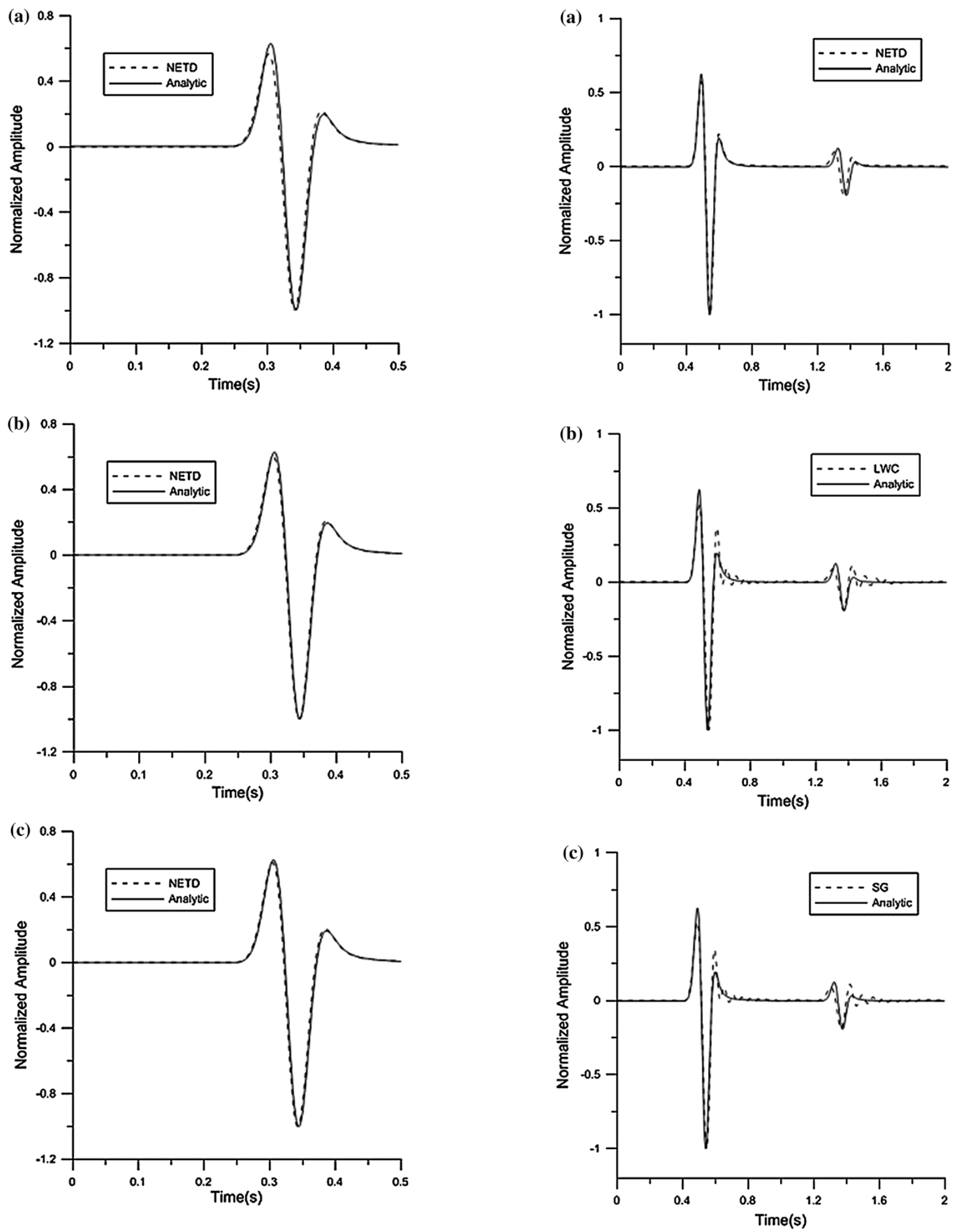

Fig. 6 Comparison of waveforms for the displacement generated by the NETD and the analytic method for the acoustic wave equation in the homogeneous medium. The spatial and temporal increments are a $h=\Delta x=\Delta z=40 \mathrm{~m}$ and $\Delta t=0.001 \mathrm{~s}, \mathbf{b} h=\Delta x=\Delta z=40 \mathrm{~m}$ and $\Delta t=5 \times 10^{-4} \mathrm{~s}$, and $\mathbf{c} h=\Delta x=\Delta z=20 \mathrm{~m}$ and $\Delta t=2.5 \times 10^{-4} \mathrm{~s}$, respectively

Fig. 7 Comparisons of waveforms generated by numerical schemes on the grids $(\Delta x=\Delta z=40 \mathrm{~m})$ and the analytic method for the twolayer acoustic model. The numerical waveforms are generated by $\mathbf{a}$ the NETD method, $\mathbf{b}$ the fourth-order LWC method, and $\mathbf{c}$ the fourth-order SG method, respectively 

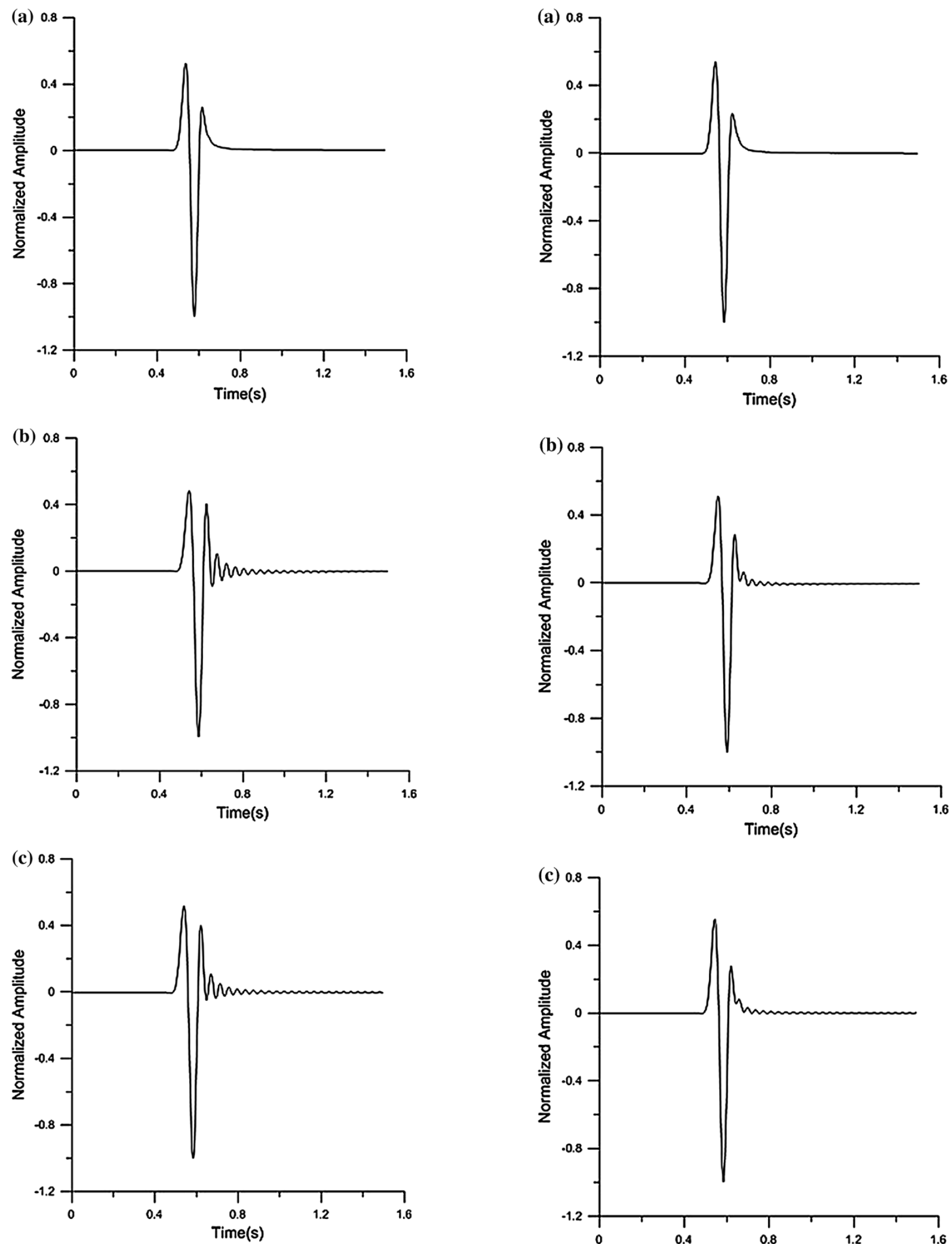

Fig. 8 The waveforms generated by a the NETD method, b the fourth-order LWC method, and $\mathbf{c}$ the fourth-order SG method for the 2-D homogeneous medium at receiver $\mathrm{R}_{1}$

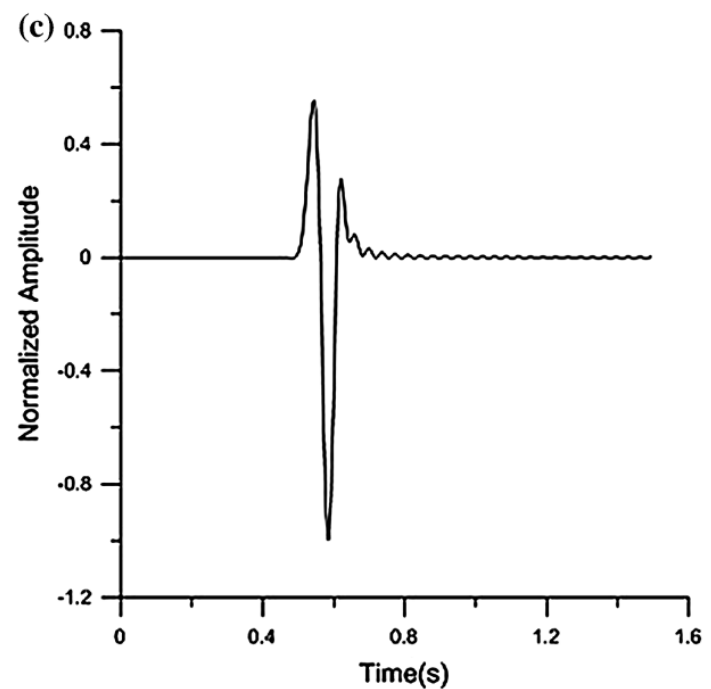

Fig. 9 The waveforms generated by a the NETD method, b the fourth-order LWC method, and $\mathbf{c}$ the fourth-order SG method for the 2-D homogeneous medium at receiver $\mathrm{R}_{2}$ 


\section{Numerical dispersion and efficiency}

To investigate the validity of suppressing the numerical dispersion and the computational efficiency of the NETD method, we consider the acoustic wave equation in a homogeneous medium with a velocity of $c_{0}=4 \mathrm{~km} / \mathrm{s}$. In the first example, we choose the computational domain of $0 \leq x \leq 20 \mathrm{~km}$ and $0 \leq z \leq 20 \mathrm{~km}$ and a uniform spatial grid ( $h=\Delta x=\Delta z=50 \mathrm{~m}$ ) is used. The source with a frequency of $f_{0}=20 \mathrm{~Hz}$ is located at the center of the computational domain, whose time variation is given by Eq. (30). The receivers $R_{1}$ and $R_{2}$ are located at $(x=12 \mathrm{~km}, \mathrm{z}=10 \mathrm{~km})$ and $(x=11 \mathrm{~km}, \mathrm{z}=11.73 \mathrm{~km})$, respectively. In the experiment, we compared the waveforms generated by the NETD, the fourth-order LWC, and the fourth-order SG at receiver $R_{1}$ and $R_{2}$.

Figure $8 \mathrm{a}-\mathrm{c}$ shows the waveforms generated by the NETD, the fourth-order LWC, and the fourth-order SG at receiver $\mathrm{R}_{1}$, respectively, and Fig. 9a-c shows the waveforms generated by the NETD, the fourth-order LWC, and the fourth-order SG at receiver $R_{2}$, respectively. The figures do not show obvious numerical dispersion from the waveforms generated by NETD at either receiver, whereas the waveforms generated by the other two methods do suggest numerical dispersion. This indicates that the NETD effectively suppresses numerical dispersion as compared with the other two methods, when coarse grids (four sampling grid points per wavelength) are used. In addition, the waveforms generated by NETD at receiver $R_{1}$ are identical to the waveforms at receiver $R_{2}$, whereas both the waveforms generated by the other two methods at receiver $R_{1}$ show more serious dispersion than waveforms at receiver $R_{2}$. This demonstrates that the NETD has less numerical dispersion anisotropy than the other methods as noted in Sect. 6.

In the second example, we compare the computational efficiency and cost of NETD with those of LWC and SG. In this experiment, the computational domain chosen is $0 \leq x \leq 10 \mathrm{~km}$ and $0 \leq z \leq 10 \mathrm{~km}$. The source is still located at the center of this domain and the other computational parameters are the same as in the last example. Figure 10ac shows the wave field snapshots at $T=1.0 \mathrm{~s}$ on the coarse spatial grid condition $(\Delta x=\Delta z=50 \mathrm{~m})$ of the NETD, the fourth-order LWC, and the SG, respectively. Restricted by the stability condition, the temporal grid size is chosen as $\Delta t=0.002 \mathrm{~s}$. Figure 10a shows a clean result, whereas Fig. 10b, c shows serious numerical dispersion. To avoid numerical dispersion in the results from LWC and SG, we use finer grids $(h=\Delta x=\Delta z=20 \mathrm{~m})$. Figure 11a, b shows the wave field snapshots at $T=1.0 \mathrm{~s}$ of LWC and SG on the fine grids, in which the same Courant number in the computation shown in Fig. 10a is used. By comparing Figs. 10a and 11, we find that the NETD method on the coarse grids can produce a result that is comparable with those of LWC and SG on much finer girds. As a result, the computational cost of generating a comparably accurate result using NETD is much less than the costs associated with the other two methods. In this example, NETD took about $26 \mathrm{~s}$ to compute Fig. 10a, whereas LWC and SG spend about 65 and $68 \mathrm{~s}$ on generating Fig. 11a and b, respectively. This means that NETD is about 2.5 times faster and 2.62 times faster than LWC and SG, respectively. In addition, NETD requires less computational storage. For example, NETD requires 18 arrays to store all the computational information needed in the method and the numbers for the grid points of each array are $201 \times 201$ on coarse grids. The fourth-order LWC and SG need only three and five arrays, respectively. But to avoid numerical dispersion, a finer grid is needed for both LWC and SG. As a result, the numbers of the grid points of LWC and SG are both $501 \times 501$ (10 spatial grid points per wavelength). Therefore, NETD requires only $96.58 \%$ of the storage required by LWC and requires only $57.95 \%$ of the storage required by $\mathrm{SG}$.

\section{Numerical simulations}

In this section, we use our proposed method to simulate the seismic wave propagating in multilayer media with strong velocity contrasts, a complex geological medium, and heterogeneous media in order to further demonstrate the validity of the NETD method.

\subsection{Three-layer model}

In the first example, we choose a three-layer model with a computational domain of $0 \leq x \leq 6 \mathrm{~km}$ and $0 \leq z \leq 6 \mathrm{~km}$ as shown in Fig. 12. The velocity is $3.0 \mathrm{~km} / \mathrm{s}$ in the top layer, $2.0 \mathrm{~km} / \mathrm{s}$ in the middle thin layer, and $4.0 \mathrm{~km} / \mathrm{s}$ in the bottom layer. There are strong velocity contrasts between the adjacent layers. We locate the same explosive source given by Eq. (30) with a frequency of $f_{0}=25 \mathrm{~Hz}$ at the center of the domain. The spatial and temporal increments are chosen as $\Delta x=\Delta z=20 \mathrm{~m}$ (four sampling grid points per minimum wavelength) and $\Delta t=5 \times 10^{-4} \mathrm{~s}$, respectively.

Figure 13 shows the wave field snapshots generated by NETD at time $T=0.3 \mathrm{~s}, T=0.5 \mathrm{~s}, T=0.8 \mathrm{~s}$, and $T=1.0 \mathrm{~s}$. There are no reflection waves in the snapshot at time $T=0.3 \mathrm{~s}$, as the acoustic wave propagates in the middle layer (an isotropic homogeneous medium). The snapshots at $T=0.5 \mathrm{~s}, T=0.8 \mathrm{~s}$, and $T=1.0 \mathrm{~s}$ show that the reflected and transmitted waves propagate from the upper and bottom interfaces. In short, the four snapshots in 

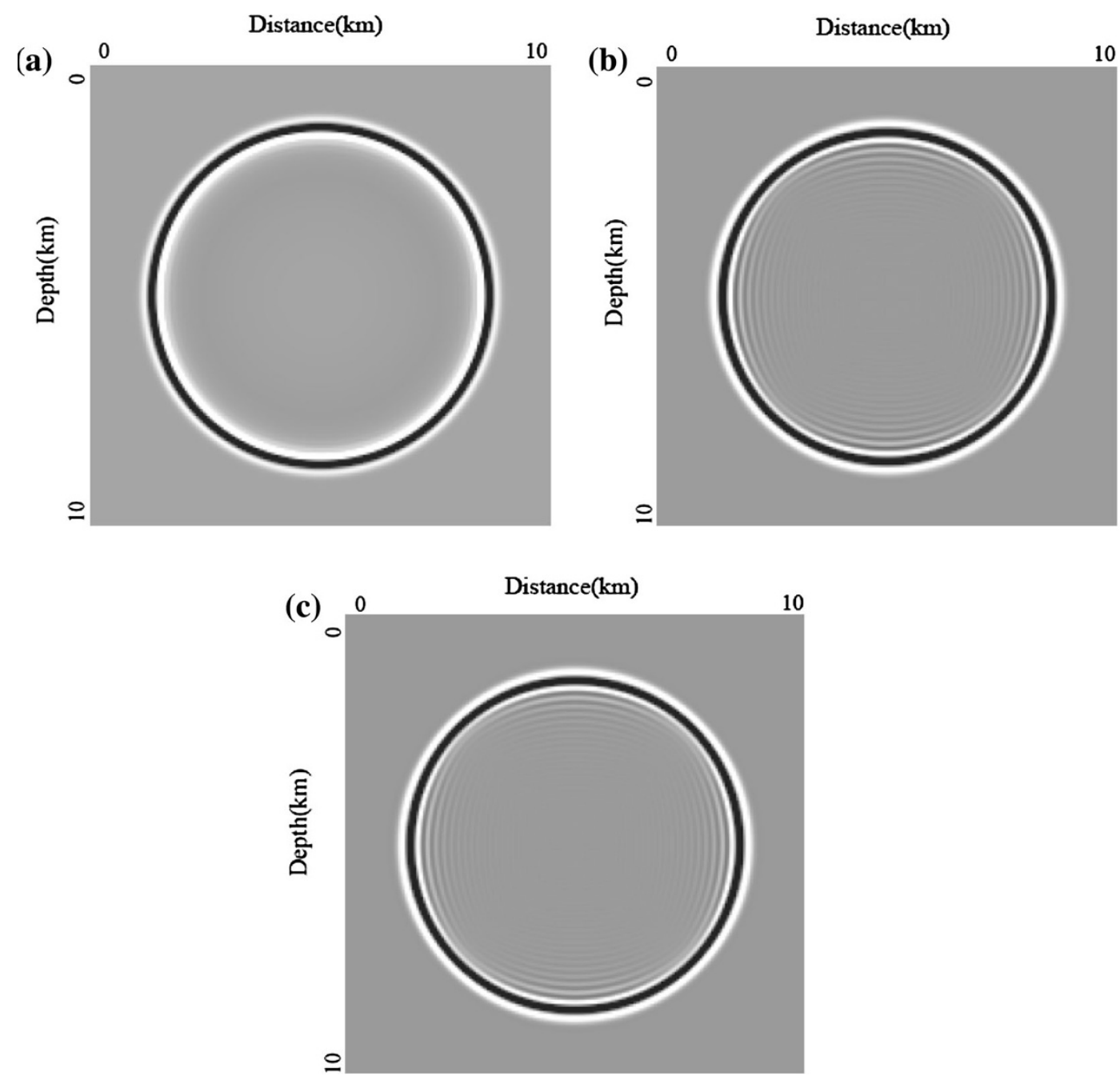

Fig. 10 Snapshots of acoustic wave field at $T=1.0 \mathrm{~s}$ on the coarse grid ( $\Delta x=\Delta z=50 \mathrm{~m}$ ) generated by a the NETD method, $\mathbf{b}$ the fourth-order LWC method, and $\mathbf{c}$ the fourth-order SG method, respectively
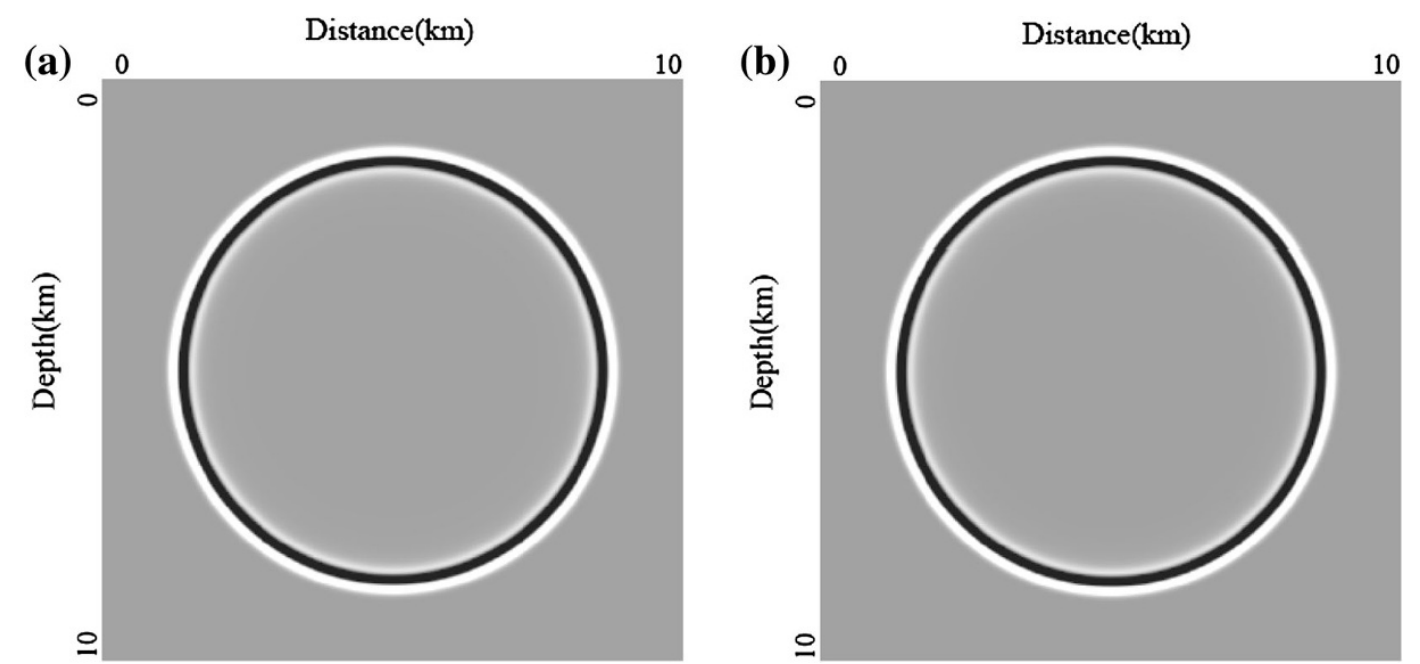

Fig. 11 Snapshots of acoustic wave field at $T=1.0 \mathrm{~s}$ on the fine grid ( $\Delta x=\Delta z=20 \mathrm{~m}$ ), generated by a the fourth-order LWC method and b the fourth-order SG method 


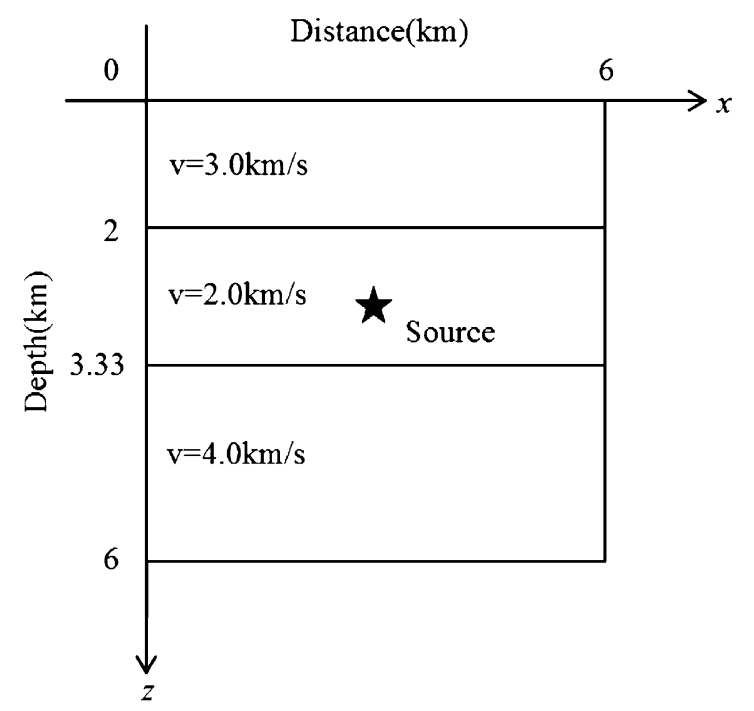

Fig. 12 The three-layer model with a size of $0 \leq x \leq 6 \mathrm{~km}$, $0 \leq z \leq 6 \mathrm{~km}$ and the two strong inner horizontal interfaces at a depth of $z=2.0 \mathrm{~km}$ and $z=3.33 \mathrm{~km}$. The source is located at the center of the model. The respective velocity for each layer is $3.0,2.0$, and $4.0 \mathrm{~km} / \mathrm{s}$
Fig. 13 clearly show the wave propagation phenomena including reflection and transmission at the interfaces without visible numerical dispersion. Figure 14 shows the wave field snapshots generated by the fourth-order LWC at time $T=1.0 \mathrm{~s}$ for the same computational parameters as those shown in Fig. 13. The snapshot in Fig. 14 shows serious numerical dispersion. The comparison in this experiment demonstrates that NETD can better deal with the models with strong velocity between the adjacent layers on coarse spatial grids than LWC.

\subsection{Corner-edge model}

In the second experiment, we consider a quite complex model. The corner-edge model consisting of three domains (I, II, and III) is shown in Fig. 15 . The velocity is $2.0 \mathrm{~km} / \mathrm{s}$ in domain I, $3.0 \mathrm{~km} / \mathrm{s}$ in domain II, and $4.0 \mathrm{~km} / \mathrm{s}$ in domain III, respectively. The size of the entire domain is $0 \leq x \leq 12 \mathrm{~km}$ and $0 \leq z \leq 12 \mathrm{~km}$, and the same source as
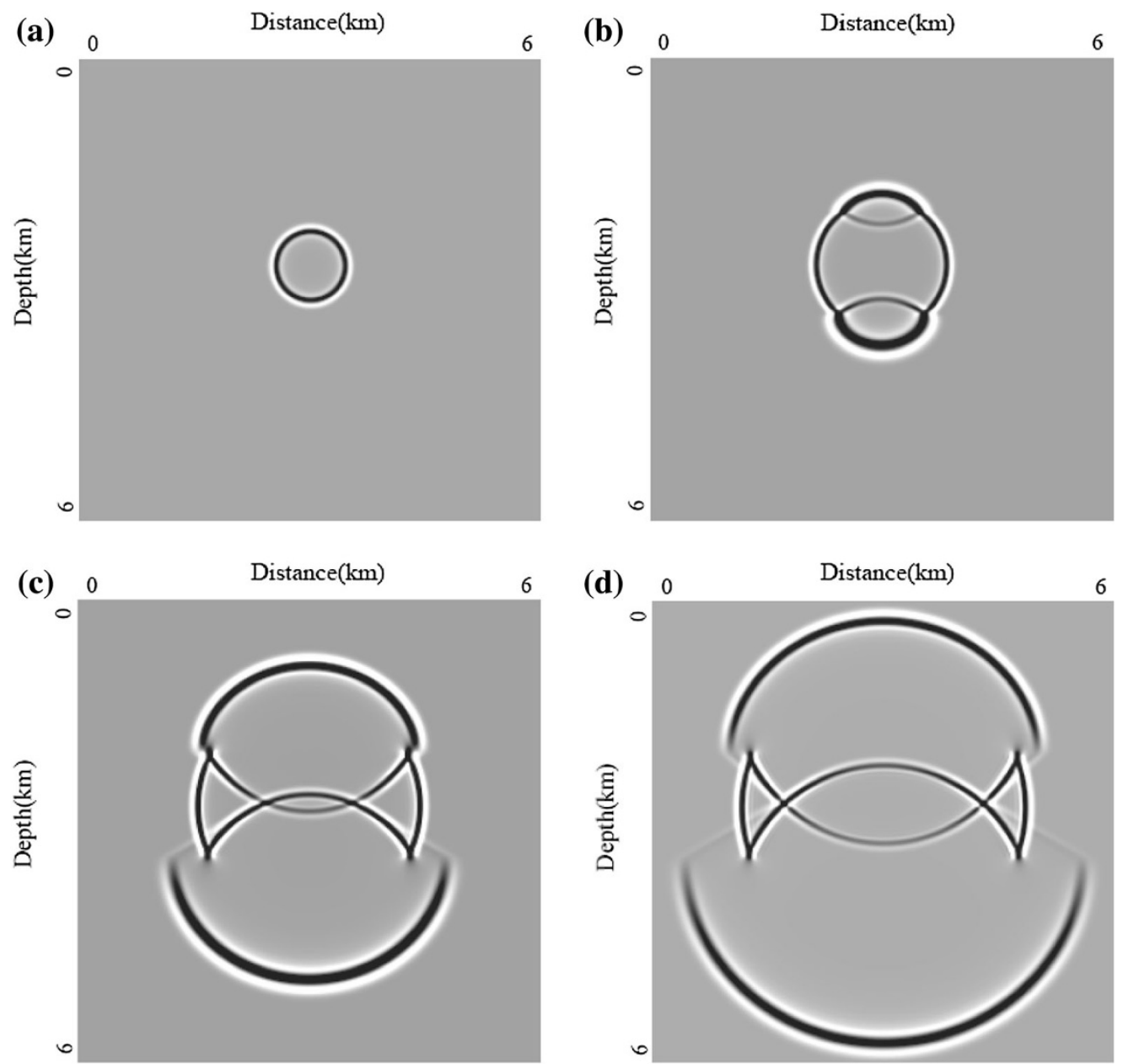

Fig. 13 Snapshots of acoustic wave fields at time a $0.3 \mathrm{~s}, \mathbf{b} 0.5 \mathrm{~s}, \mathbf{c} 0.8 \mathrm{~s}$, and $\mathbf{d} 1.0 \mathrm{~s}$ for the three-layer model generated by the NETD method 


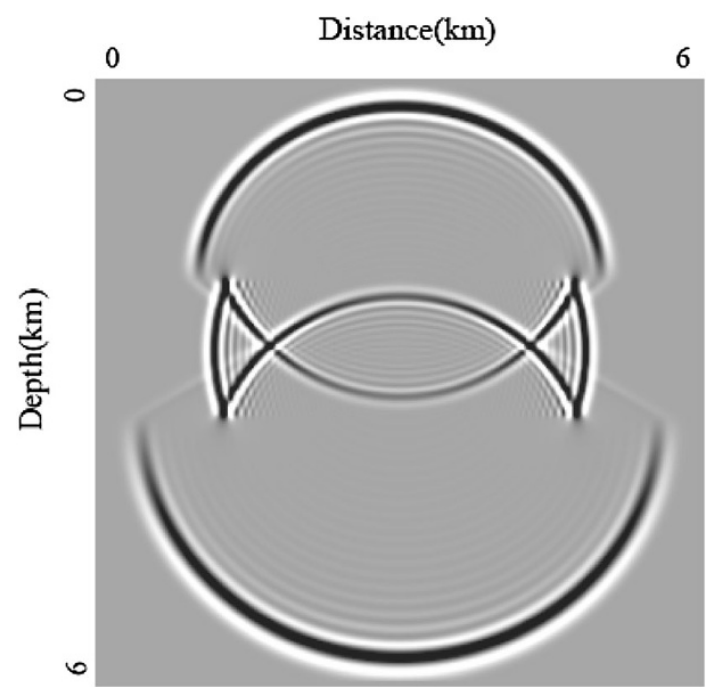

Fig. 14 Snapshots of acoustic wave fields at $1.0 \mathrm{~s}$ for the three-layer model generated by the LWC method

the previous examples with a frequency of $f_{0}=20 \mathrm{~Hz}$ located at the center of the model is used. The chosen spatial increment is $\Delta x=\Delta z=30 \mathrm{~m}$ and the chosen temporal increment is $\Delta t=7.5 \times 10^{-4} \mathrm{~s}$. Thus, there are five sampling grid points per minimum wavelength.

Figure 16 shows the wave field snapshots generated by the NETD at time $T=0.5 \mathrm{~s}, T=1.0 \mathrm{~s}$ and $T=1.5 \mathrm{~s}$. The snapshots show complex reflected and transmitted waves from the horizontal inner interface and the vertical inner interface without visible numerical dispersion. This demonstrates that the NETD can effectively simulate wave propagation in a quite complex geological case.

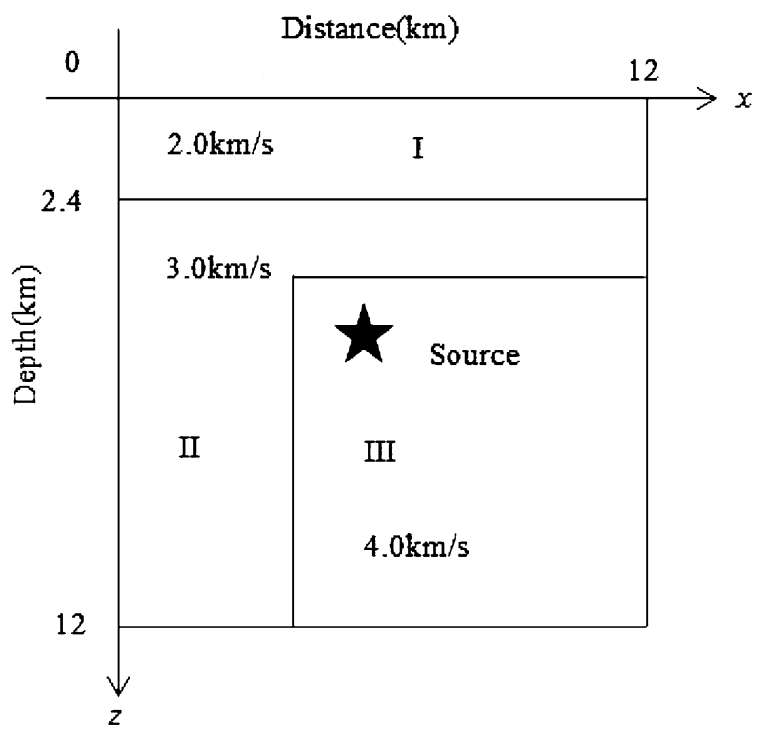

Fig. 15 The corner-edge velocity model

\subsection{Marmousi model}

In the following example, we choose the heterogeneous Marmousi model (Versteeg and Grau 1991) shown in Fig. 17. In the experiment, the number of grid pints is $384 \times 122$ and the spatial and temporal increments are $\Delta x=\Delta z=24 \mathrm{~m}$ and $\Delta t=4.36 \times 10^{-4} \mathrm{~s}$, respectively. The source, which has a frequency of $f_{0}=15 \mathrm{~Hz}$, is located in the middle on the surface. The maximal acoustic velocity of this model is $5.5 \mathrm{~km} / \mathrm{s}$, so there are four grid points in a minimal wavelength.

Figure 18 shows the wave field snapshots at time $T=0.6 \mathrm{~s}, T=0.9 \mathrm{~s}, T=1.2 \mathrm{~s}$, , and $T=1.5 \mathrm{~s}$. The perfectly matched layer absorbing boundary condition (Dimitri and Jeroen 2003; Frank et al. 1996) is used in this experiment. There is no visible numerical dispersion in the four wave field snapshots. Figure 19 shows the synthetic seismogram on the surface generated by the NETD. The seismogram is recorded at time $T=3.0 \mathrm{~s}$ by 122 receivers with a space interval of $24 \mathrm{~m}$ apart on the surface. It is known that Marmousi model is widely used as a performance measure for various methods. The numerical results indicate that NETD can effectively simulate wave propagation in an extremely complex case.

\subsection{SEG model}

In the last example for the acoustic wave, we choose the SEG model (Fig. 20) to test the performance of NETD. In the experiment, the number of mesh points is $400 \times 201$, and the chosen space and time step are $\Delta x=\Delta z=10 \mathrm{~m}$ and $\Delta t=2.0 \times 10^{-4} \mathrm{~s}$, respectively. We locate the same explosive source as the previous experiments with a frequency of $f_{0}=30 \mathrm{~Hz}$ in the middle of the surface.

Figure 21 shows the wave field snapshots at time $T=$ $0.3 \mathrm{~s}, T=0.5 \mathrm{~s}, T=0.8 \mathrm{~s}, T=1.0 \mathrm{~s}$, and $T=1.2 \mathrm{~s}$. The perfectly matched layer absorbing boundary condition is used in this experiment. We can see that the five wave field snapshots show no visible numerical dispersion. The synthetic seismogram in Fig. 22 generated by the NETD method is recorded at time $T=1.6 \mathrm{~s}$ by 201 receivers on the surface. We can see that Fig. 22 shows a clear waveform and the different reflected waves from different interior interfaces are evident. For example, $R 1, R 2, R 3$, and $R 4$ are reflected waves from the nearly horizontal interfaces $D 1, D 2, D 3$, and $D 4$, respectively. The numerical results further indicate that the NETD can be used to simulate the wave propagation in a complex medium. In addition, the snapshots demonstrate that the NETD method can be combined with the perfectly matched layer absorbing boundary condition successfully and effectively. 

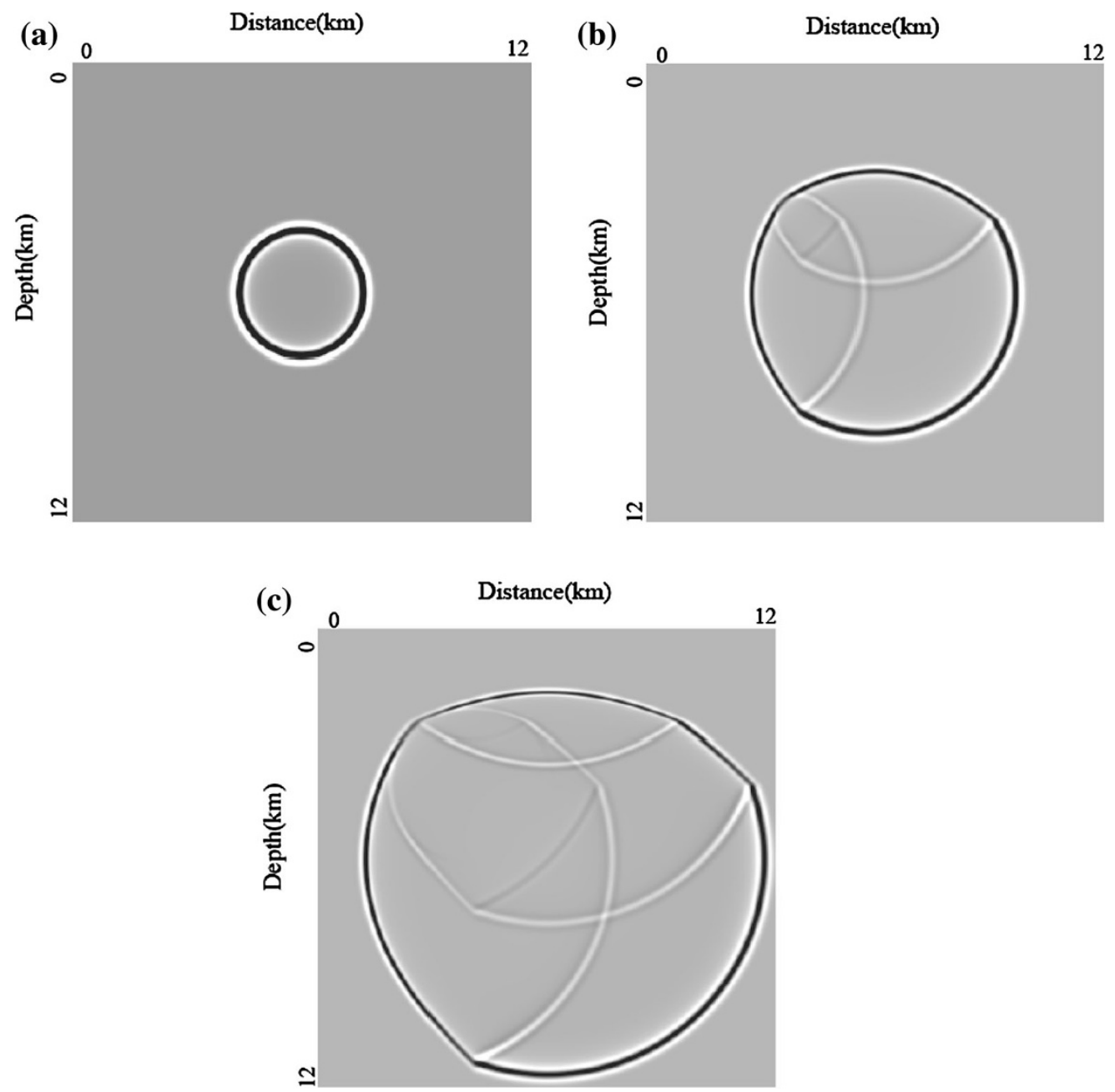

Fig. 16 Snapshots of acoustic wave fields at time a $0.5 \mathrm{~s}, \mathrm{~b} 1.0 \mathrm{~s}$, and c $1.5 \mathrm{~s}$ for the corner-edge model generated by the NETD method

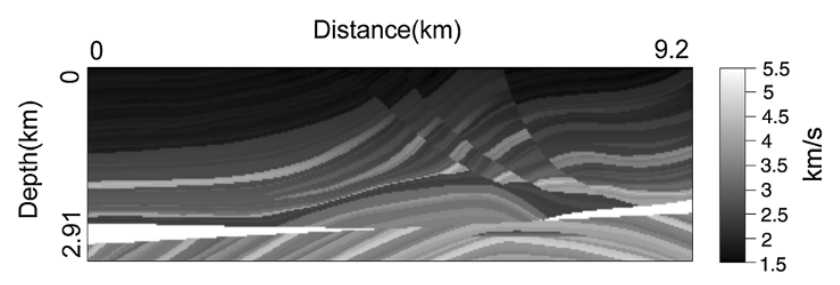

Fig. 17 The Marmousi model

\subsection{Homogeneous elastic model}

For the elastic case, first we consider the following elastic wave equations in a $2 \mathrm{D}$ homogeneous TI medium:

$$
\left\{\begin{array}{l}
\rho \frac{\partial^{2} u}{\partial t^{2}}=c_{11} \frac{\partial^{2} u}{\partial x^{2}}+\left(c_{13}+c_{44}\right) \frac{\partial^{2} w}{\partial x \partial z}+c_{44} \frac{\partial^{2} u}{\partial z^{2}}+f_{1} \\
\rho \frac{\partial^{2} w}{\partial t^{2}}=c_{44} \frac{\partial^{2} w}{\partial x^{2}}+\left(c_{13}+c_{44}\right) \frac{\partial^{2} u}{\partial x \partial z}+c_{33} \frac{\partial^{2} w}{\partial z^{2}}+f_{2}
\end{array}\right.
$$

where $u$ and $w$ are the displacement components in the $x$ - and $z$-directions, respectively. $c_{11}, c_{13}, c_{33}$, and $c_{44}$ are the elastic constants; $\rho$ is the medium density; and $f_{1}$ and $f_{2}$ are the force source components in the $x$ - and $z$ directions.

In this example, the chosen computational parameters are $c_{11}=45, c_{13}=9.6, c_{33}=37.5, c_{44}=12 \mathrm{GPa}$, and $\rho=1.0$ $\mathrm{g} / \mathrm{cm}^{3}$. The number of mesh points is $401 \times 401$, the spatial grid increment is $\Delta x=\Delta z=30 \mathrm{~m}$, and the time increment is $t=6.7 \times 10^{-4} \mathrm{~s}$. The source located at the center of the computational domain is a Ricker wavelet same as the previous experiments, which has a frequency of $f_{0}=30 \mathrm{~Hz}$.

Figure $23 \mathrm{a}, \mathrm{b}$ shows the $x$ - and $z$-component snapshots at $T=0.6 \mathrm{~s}$ generated by the NETD method, respectively. The snapshots show clear wave field information of the displacement without visible numerical dispersion. In addition, we can see clearly the cusps and the anisotropy of the velocity of the wave propagating from Fig. 23 . In short, the numerical results demonstrate that the 

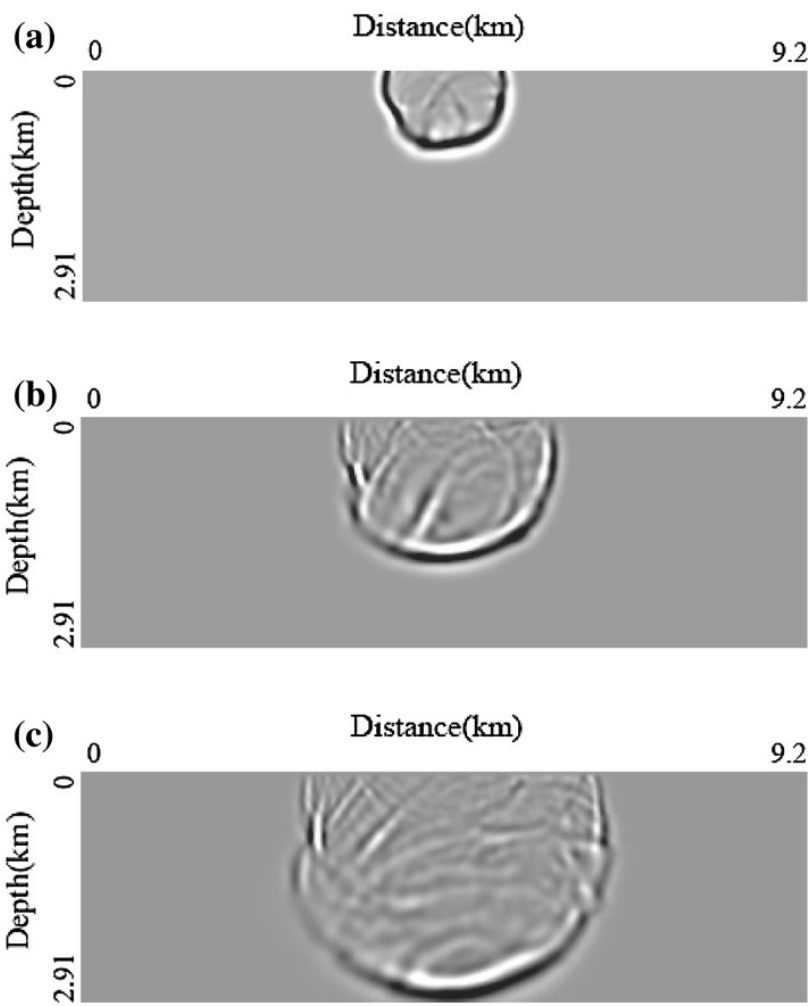

(d) Distance(km)

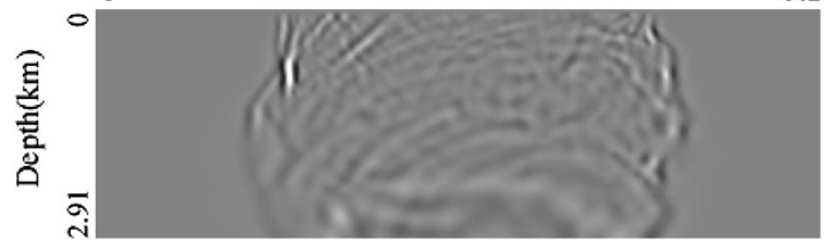

Fig. 18 Snapshots of acoustic wave fields at time a $0.6 \mathrm{~s}$, b $0.9 \mathrm{~s}$, c $1.2 \mathrm{~s}$, and $\mathbf{d} 1.5 \mathrm{~s}$ for the Marmousi model generated by the NETD method

NETD method can simulate the elastic wave propagation effectively.

\subsection{Two-layer elastic model}

In the last elastic example, we choose a two-layer model with large velocity contrasts across the interface. The size of the computational domain is $0 \leq x \leq 4 \mathrm{~km}$ and $0 \leq z \leq 4 \mathrm{~km}$, and we locate the horizontal strong interface at depth of $2.4 \mathrm{~km}$. The Lame constants and densities of the two-layer model are $\rho_{1}=1.5 \mathrm{~g} / \mathrm{cm}^{3}, \lambda_{1}=1.5 \mathrm{GPa}$, $\mu_{1}=2.5 \mathrm{GPa}$ in the top layer and $\rho_{2}=2.0 \mathrm{~g} / \mathrm{cm}^{3}$, $\lambda_{2}=11.0 \mathrm{GPa}, \mu_{2}=15.0 \mathrm{GPa}$ in the bottom layer. These computational parameters correspond to the $\mathrm{P}$ - and SVwave velocities of 1.63 and $1.29 \mathrm{~km} / \mathrm{s}$ in the top layer, and

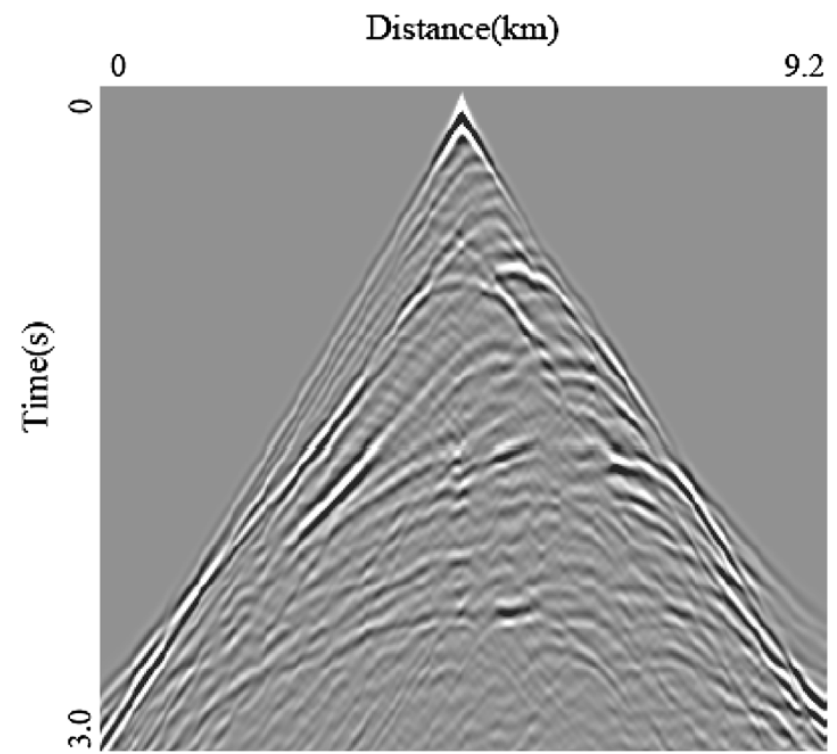

Fig. 19 The synthetic seismogram on the surface, generated by the NETD method for the Marmousi model

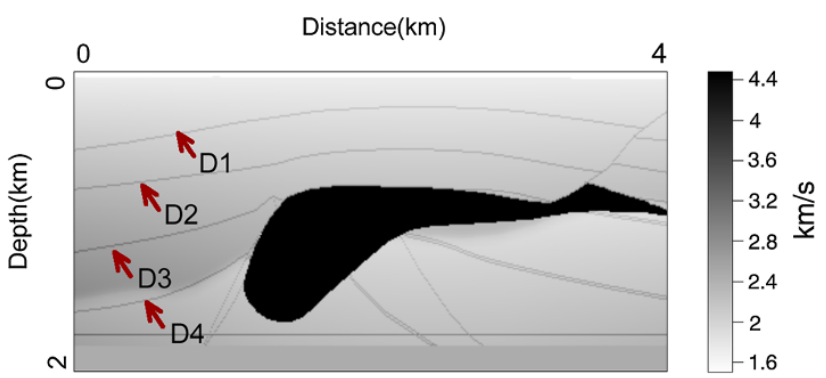

Fig. 20 The SEG model

3.61 and $2.74 \mathrm{~km} / \mathrm{s}$ in the bottom layer. The chosen spatial increment is $\Delta x=\Delta z=15 \mathrm{~m}$, and the chosen temporal increment is $t=5 \times 10^{-4} \mathrm{~s}$. The source, which has a frequency of $f_{0}=20 \mathrm{~Hz}$, is a symmetric Ricker wavelet located at ( 2 and $1.85 \mathrm{~km})$. Figure $24 \mathrm{a}, \mathrm{b}$ shows the $x$ direction and $z$-direction snapshots at $T=0.6 \mathrm{~s}$, generated by the NETD. The numerical results clearly show the elastic wave propagation phenomena in the two-layer model, which indicates that the NETD can also be used in a multilayer medium with strong interfaces for elastic wave cases.

\section{Conclusions}

In this paper, we proposed a new NETD method for solving seismic wave equations and investigated in detail the properties of the NETD method, including its accuracy, stability, numerical dispersion, and computational efficiency. Both 

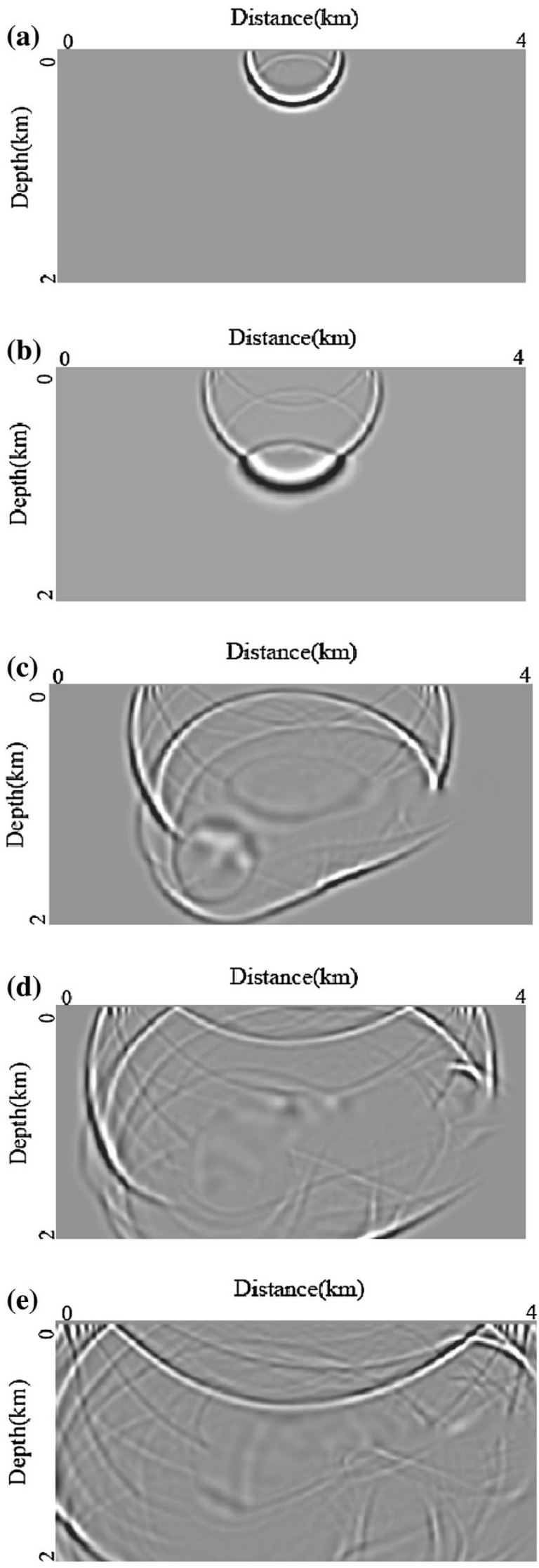

4Fig. 21 Snapshots of acoustic wave fields at time a $0.3 \mathrm{~s}, \mathbf{b} 0.5 \mathrm{~s}$, c $0.8 \mathrm{~s}$, d $1.0 \mathrm{~s}$, and e $1.2 \mathrm{~s}$ for the SEG model generated by the NETD method

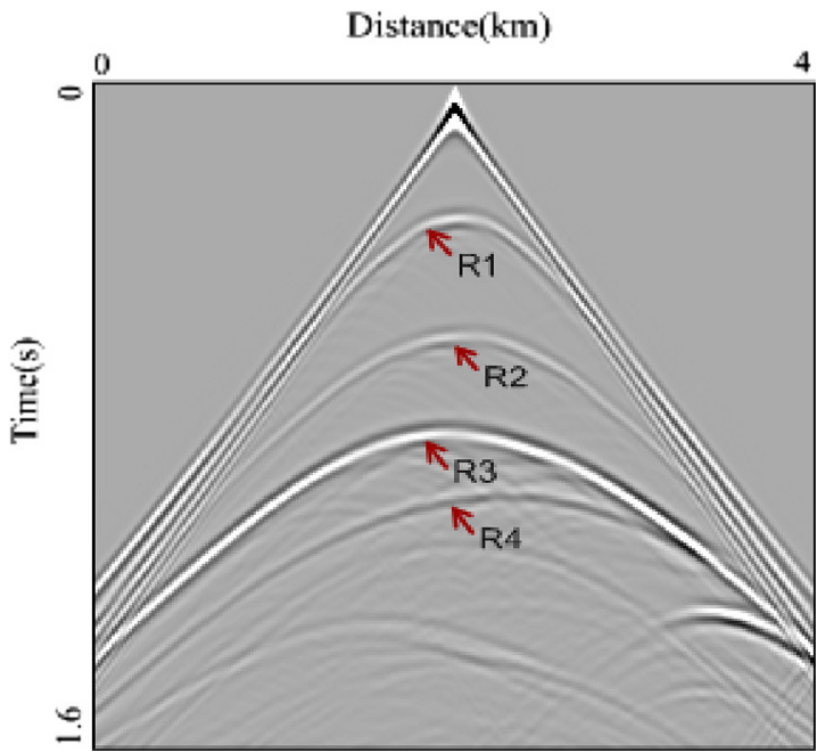

Fig. 22 The synthetic seismogram on the surface generated by the NETD method for the SEG model

theoretical analyses and numerical experiments show that the NETD method has the fourth-order accuracy in space and the second-order accuracy in time. The numerical experiments also show that the NETD method can be widely used in seismic modeling from acoustic/elastic homogeneous; multilayer and complex heterogeneous models such as the Marmousi and SEG models.

The proposed method is based on the idea of the Lie group method, and it belongs to the category of integration method in that it preserves qualitative properties of the ODEs, so the NETD provides more accurate results than LWC and SG. For example, the relative error of NETD is much smaller than those of LWC and SG methods. Furthermore, because of the simultaneous use of wave displacement, particle velocity, and their respective gradients to reconstruct the wave displacement and velocity fields, the method effectively suppresses the numerical dispersion when coarse spatial grid is used and when there are strong velocity contrasts across the interface in a complex medium. As a result, the NETD has high computational efficiency and low memory requirement as compared with LWC and SG. However, the stability criterion of the NETD method is relatively compact. It needs to be further improved in the future. 

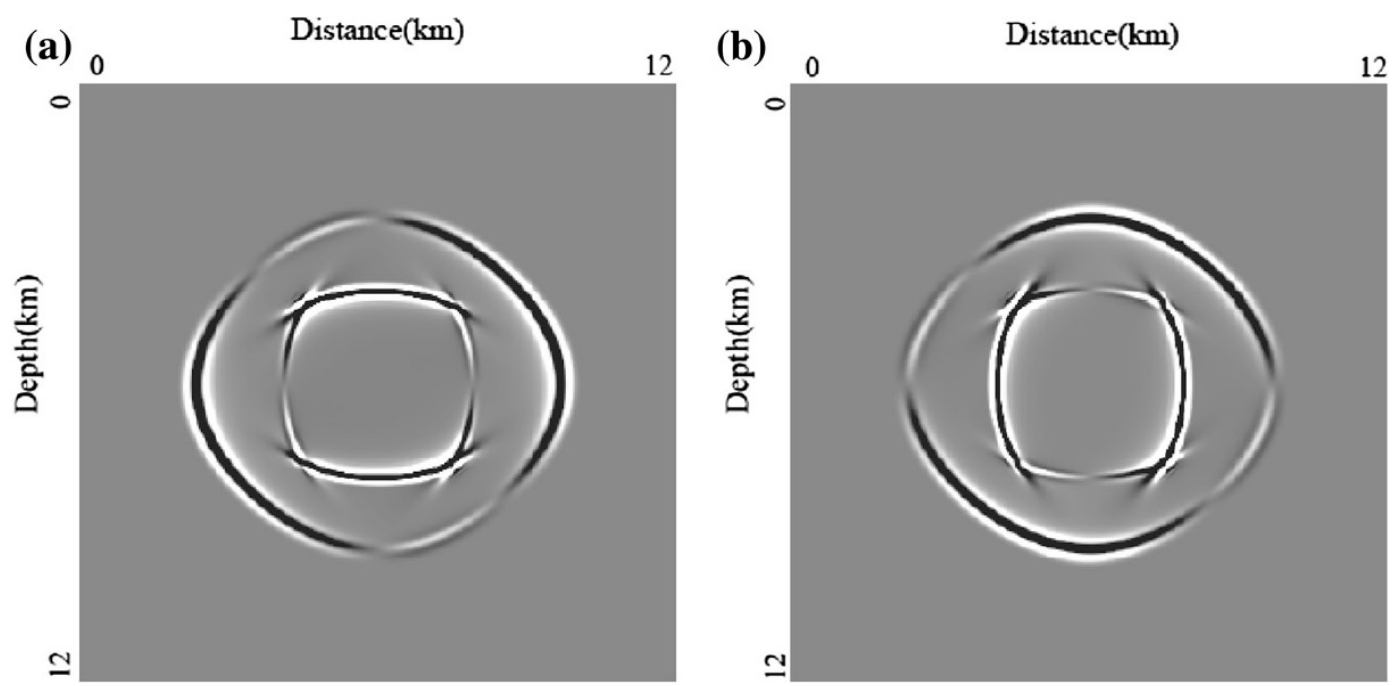

Fig. 23 Snapshots of the elastic wave field in the 2-D homogeneous TI medium at time $0.6 \mathrm{~s}$ generated by the NETD method: a the $x$-direction component and $\mathbf{b}$ the $z$-direction component
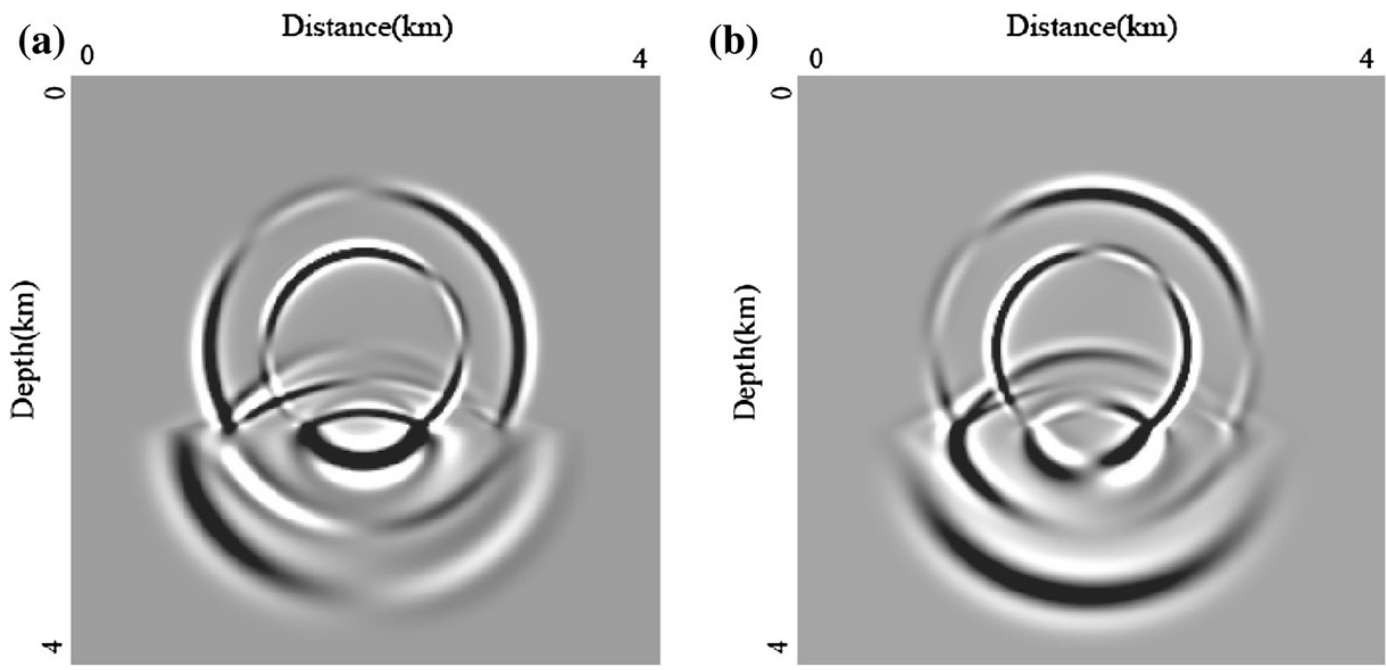

Fig. 24 Snapshots of the $x$-direction and the $z$-direction components for the two-layer elastic model at time $0.6 \mathrm{~s}$ generated by the NETD method

Acknowledgments The study was supported by the National Natural Science Foundation of China (Grant No. 41230210) and by the Statoil Company (Contract No. 4502502663).

\section{Appendix 1: Approximations of high-order spatial derivatives}

To compute the high-order spatial derivatives in the wave equations (12) and (21), we follow Tong et al. (2011) and Yang et al. $(2006,2007)$ and obtain the approximation formulae. For convenience, we list the formulae used in the NETD method as follows:

$$
\begin{aligned}
\left(\frac{\partial^{2} u}{\partial x^{2}}\right)_{i, j}^{n}= & \frac{2}{\Delta x^{2}}\left(u_{i+1, j}^{n}-2 u_{i, j}^{n}+u_{i-1, j}^{n}\right) \\
& -\frac{1}{2 \Delta x}\left(\left(\frac{\partial u}{\partial x}\right)_{i+1, j}^{n}-\left(\frac{\partial u}{\partial x}\right)_{i-1, j}^{n}\right)
\end{aligned}
$$

$$
\begin{aligned}
\left(\frac{\partial^{2} u}{\partial z^{2}}\right)_{i, j}^{n}= & \frac{2}{\Delta z^{2}}\left(u_{i, j+1}^{n}-2 u_{i, j}^{n}+u_{i, j-1}^{n}\right) \\
& -\frac{1}{2 \Delta z}\left(\left(\frac{\partial u}{\partial z}\right)_{i, j+1}^{n}-\left(\frac{\partial u}{\partial z}\right)_{i, j-1}^{n}\right)
\end{aligned}
$$




$$
\begin{aligned}
\left(\frac{\partial^{2} u}{\partial x \partial z}\right)_{i, j}^{n}= & \frac{1}{2 \Delta x \Delta z}\left(u_{i+1, j+1}^{n}+u_{i-1, j-1}^{n}-u_{i-1, j+1}^{n}-u_{i+1, j-1}^{n}\right) \\
& -\frac{1}{8 \Delta z}\left(\left(\frac{\partial u}{\partial x}\right)_{i+1, j+1}^{n}-\left(\frac{\partial u}{\partial x}\right)_{i-1, j-1}^{n}+\left(\frac{\partial u}{\partial x}\right)_{i-1, j+1}^{n}\right. \\
& \left.-\left(\frac{\partial u}{\partial x}\right)_{i+1, j-1}^{n}\right)-\frac{1}{8 \Delta x}\left(\left(\frac{\partial u}{\partial z}\right)_{i+1, j+1}^{n}\right. \\
& \left.-\left(\frac{\partial u}{\partial z}\right)_{i-1, j-1}^{n}\left(\frac{\partial u}{\partial z}\right)_{i+1, j-1}^{n}-\left(\frac{\partial u}{\partial z}\right)_{i-1, j+1}^{n}\right)
\end{aligned}
$$

$$
\begin{aligned}
\left(\frac{\partial^{3} u}{\partial x^{3}}\right)_{i, j}^{n}= & \frac{15}{2 \Delta x^{3}}\left(u_{i+1, j}^{n}-u_{i-1, j}^{n}\right) \\
& -\frac{3}{2 \Delta x^{2}}\left(\left(\frac{\partial u}{\partial x}\right)_{i+1, j}^{n}+8\left(\frac{\partial u}{\partial x}\right)_{i, j}^{n}+\left(\frac{\partial u}{\partial x}\right)_{i-1, j}^{n}\right)
\end{aligned}
$$$$
\left(\frac{\partial^{3} u}{\partial z^{3}}\right)_{i, j}^{n}=\frac{15}{2 \Delta z^{3}}\left(u_{i, j+1}^{n}-u_{i, j-1}^{n}\right)
$$$$
-\frac{3}{2 \Delta x^{2}}\left(\left(\frac{\partial u}{\partial z}\right)_{i, j+1}^{n}+8\left(\frac{\partial u}{\partial z}\right)_{i, j}^{n}+\left(\frac{\partial u}{\partial z}\right)_{i, j-1}^{n}\right) \text {, }
$$

$$
\begin{aligned}
\left(\frac{\partial^{3} u}{\partial x^{2} \partial z}\right)_{i, j}^{n}= & \frac{5}{4 \Delta x^{2} \Delta z}\left(u_{i+1, j+1}^{n}-u_{i-1, j-1}^{n}+u_{i-1, j+1}^{n}\right. \\
& \left.-u_{i+1, j-1}^{n}+2 u_{i, j-1}^{n}-2 u_{i, j+1}^{n}\right) \\
& -\frac{1}{4 \Delta x^{2}}\left(\left(\frac{\partial u}{\partial z}\right)_{i+1, j+1}^{n}+\left(\frac{\partial u}{\partial z}\right)_{i-1, j-1}^{n}\right. \\
& +\left(\frac{\partial u}{\partial z}\right)_{i-1, j+1}^{n}+\left(\frac{\partial u}{\partial z}\right)_{i+1, j-1}^{n}-2\left(\frac{\partial u}{\partial z}\right)_{i, j-1}^{n} \\
& \left.-2\left(\frac{\partial u}{\partial z}\right)_{i, j+1}^{n}\right)-\frac{1}{4 \Delta x \Delta z}\left(\left(\frac{\partial u}{\partial x}\right)_{i+1, j+1}^{n}\right. \\
& \left.+\left(\frac{\partial u}{\partial x}\right)_{i-1, j-1}^{n}-\left(\frac{\partial u}{\partial x}\right)_{i-1, j+1}^{n}-\left(\frac{\partial u}{\partial x}\right)_{i+1, j-1}^{n}\right)
\end{aligned}
$$

$$
\begin{aligned}
\left(\frac{\partial^{3} u}{\partial x \partial z^{2}}\right)_{i, j}^{n}= & \frac{5}{4 \Delta x \Delta z^{2}}\left(u_{i+1, j+1}^{n}-u_{i-1, j-1}^{n}+u_{i+1, j-1}^{n}\right. \\
& \left.-u_{i-1, j+1}^{n}+2 u_{i-1, j}^{n}-2 u_{i+1, j}^{n}\right) \\
& -\frac{1}{4 \Delta z^{2}}\left(\left(\frac{\partial u}{\partial x}\right)_{i+1, j+1}^{n}+\left(\frac{\partial u}{\partial x}\right)_{i-1, j-1}^{n}\right. \\
& +\left(\frac{\partial u}{\partial x}\right)_{i+1, j-1}^{n}+\left(\frac{\partial u}{\partial x}\right)_{i-1, j+1}^{n}-2\left(\frac{\partial u}{\partial x}\right)_{i+1, j}^{n}
\end{aligned}
$$

$$
\begin{aligned}
& \left.-2\left(\frac{\partial u}{\partial x}\right)_{i-1, j}^{n}\right)-\frac{1}{4 \Delta x \Delta z}\left(\left(\frac{\partial u}{\partial z}\right)_{i+1, j+1}^{n}\right. \\
& \left.+\left(\frac{\partial u}{\partial z}\right)_{i-1, j-1}^{n}-\left(\frac{\partial u}{\partial z}\right)_{i+1, j-1}^{n}-\left(\frac{\partial u}{\partial z}\right)_{i-1, j+1}^{n}\right)
\end{aligned}
$$

where $u$ denotes the displacement.

\section{Appendix 2: Stability condition of NETD}

We use the Fourier method to obtain the stability condition of the NETD method. We consider the scalar wave equation in homogeneous media for 1-D and 2-D cases. Substituting the harmonic solution 1-D case

$\bar{V}_{j}^{n}=\left(\begin{array}{c}u^{n} \\ u_{x}^{n} \\ w^{n} \\ w_{x}^{n}\end{array}\right) \exp (i(\bar{k} j h))$,

2-D case

$\bar{V}_{j, k}^{n}=\left(\begin{array}{c}u^{n} \\ u_{x}^{n} \\ u_{z}^{n} \\ w^{n} \\ w_{x}^{n} \\ w_{z}^{n}\end{array}\right) \exp (i \bar{k} h(j \cos \theta+k \sin \theta))$,

into the NETD method, we can obtain the following equation:

$\bar{V}^{n+1}=G \bar{V}^{n}$

where $w=\frac{\partial u}{\partial t}$ and the amplification matrix $G$ is in the form

$$
\begin{aligned}
G= & \left(I-\frac{1}{2}\left(\begin{array}{cc}
\Lambda^{-2}(I-\cos (\Lambda \alpha)) M & 0 \\
\Lambda^{-2} \sin (\Lambda \alpha) M & 0
\end{array}\right)\right)^{-1} \\
& \left(\left(\begin{array}{cc}
\cos (\Lambda \alpha) & \Lambda^{-1} \sin (\Lambda \alpha) \\
-\Lambda \sin (\Lambda \alpha) & \cos (\Lambda \alpha)
\end{array}\right)\right. \\
& \left.+\frac{1}{2}\left(\begin{array}{cc}
\Lambda^{-2}(I-\cos (\Lambda \alpha)) M & 0 \\
\Lambda^{-2} \sin (\Lambda \alpha) M & 0
\end{array}\right)\right)
\end{aligned}
$$

the 1-D case:

$$
\begin{aligned}
& \Lambda=\left(\begin{array}{cc}
2 & 0 \\
0 & 2 \sqrt{3}
\end{array}\right), \\
& M=\left(\begin{array}{cc}
4 \frac{c^{2}}{h^{2}} \cos (k h) & -i \frac{c^{2}}{h} \sin (k h) \\
\frac{15 i c^{2}}{h^{3}} \sin (k h) & -\frac{3 c^{2}}{h^{2}} \cos (k h)
\end{array}\right),
\end{aligned}
$$

and the 2-D case: 


$$
\begin{aligned}
& \Lambda=\left(\begin{array}{ccc}
2 \sqrt{2} & 0 & 0 \\
0 & 2 \sqrt{3} & 0 \\
0 & 0 & 2 \sqrt{3}
\end{array}\right), \\
& M=\left(\begin{array}{ccc}
m_{11} & m_{12} & m_{13} \\
m_{21} & m_{22} & m_{23} \\
m_{31} & m_{32} & m_{33}
\end{array}\right), \\
& m_{11}=\frac{4 c^{2}}{h^{2}}(\cos (\bar{k} h \cos \theta)+\cos (\bar{k} h \sin \theta)), \\
& m_{12}=\frac{-i c^{2}}{h} \sin (\bar{k} h \cos \theta), \\
& m_{13}=\frac{-i c^{2}}{h} \sin (\bar{k} h \sin \theta), \\
& m_{21}=\frac{5 i c^{2}}{h^{3}}(2 \sin (\bar{k} h \cos \theta)+\sin (\bar{k} h \cos \theta) \cos (\bar{k} h \sin \theta)), \\
& m_{22}=-\frac{c^{2}}{h^{2}}(2 \cos (\bar{k} h \cos \theta)+\cos (\bar{k} h \cos \theta) \cos (\bar{k} h \sin \theta)), \\
& m_{23}=\frac{c^{2}}{h^{2}} \sin (\bar{k} h \cos \theta) \sin (\bar{k} h \sin \theta), \\
& m_{31}=\frac{5 i c^{2}}{h^{3}}(2 \sin (\bar{k} h \sin \theta)+\sin (\bar{k} h \sin \theta) \cos (\bar{k} h \cos \theta)), \\
& m_{33}=-\frac{c^{2}}{h^{2}}(2 \cos (\bar{k} h \sin \theta)+\cos (\bar{k} h \sin \theta) \cos (\bar{k} h \cos \theta)),
\end{aligned}
$$

where $\bar{k}$ is the wavenumber, $h=\Delta x=\Delta z$ is the spatial increment, and $\alpha$ is the Courant number defined by $\alpha=\frac{c \Delta t}{h}$.

Because of the complexity of the matrix $G$, it is difficult to analytically determine the stability condition of the method. In this paper we numerically obtain the stability condition for the 1-D and 2-D cases by solving the Eigenvalue problem $|\lambda(G)| \leq 1$. The 1-D case

$\alpha \leq \alpha_{\max } \approx 0.4232$,

and the 2-D case

$\alpha \leq \alpha_{\max } \approx 0.2583$,

where $\alpha_{\max }$ denotes the maximum courant number.

\section{Appendix 3: Derivation of 2-D dispersion relations}

To derive the numerical dispersion of the NETD scheme for the 2-D case, we consider the following harmonic solution

$$
\bar{V}_{j, k}^{n}=\left(\begin{array}{c}
u \\
u_{x} \\
u_{z} \\
w \\
w_{x} \\
w_{z}
\end{array}\right) \exp \left(i\left(n \omega_{\text {num }} \Delta t+\bar{k} h(j \cos \theta+k \sin \theta)\right)\right) .
$$

By substituting the solution into the scheme, we can obtain the numerical dispersion relation as follows:

$\operatorname{det}\left(e^{\mathrm{i} \gamma} I-G\right)=0$,

where $\gamma=\omega_{\text {num }} \Delta t, G$ is the same matrix as in Appendix 2, and $I$ is an identity matrix. From the dispersion relation (46), we can obtain the ratio of the numerical velocity to the exact velocity $c_{0}$ as follows:

$R=\frac{c_{\mathrm{num}}}{c_{0}}=\frac{\gamma}{2 \pi \alpha S_{\mathrm{p}}}$,

where $\alpha$ is the Courant number and $S_{\mathrm{p}}$ is the spatial sampling rate defined by Moszo et al. (2000).

\section{References}

Aki K, Richards PG (1980) Quantitative seismology: theory and methods. W H Freeman and Co, San Francisco

Berg P, If F, Nielsen P, Skovgaard O (1993) Diffraction by a wedge in an acoustic constant density medium. Gephys Prospect 41:803-831

Blanch JO, Robertsson JOA (1997) A modified Lax-Wendroff correction for wave propagation in media described by Zener elements. Geophys J Int 131:381-386

Booth DC, Crampin S (1983a) The anisotropic reflectivity technique: theory. Geophys J R Astron Soc 72:755-765

Booth DC, Crampin S (1983b) The anisotropic reflectivity technique: anomalous arrives from an anisotropic upper mantle. Geophys J R Astron Soc 72:767-782

Bouchon M (1996) The discrete wave number formulation of boundary integral equations and boundary element methods: a review with application to the simulations of seismic wave propagation in complex geological structures. Pure Appl Geophys $148(1-2): 3-20$

Carcione JM, Helle HB (1999) Numerical solution of the poroviscoelastic wave equation on a staggered mesh. J Comput Phys 154:520-527

Chen XF (1993) A systematic and efficient method of computing normal modes for multi-layered half-space. Geophys J Int 115:391-409

Chen S, Yang DH, Deng XY (2010) A weighted Runge-Kutta method with weak numerical dispersion for solving wave equations. Commun Comput Phys 7(5):1027-1048

Cox SM, Matthews PC (2002) Exponential time differencing for stiff systems. J Comput Phys 176:430-455

Dablain MA (1986) The application of high-order differencing to the scalar wave equation. Geophysics 51:54-66

Dimitri K, Jeroen T (2003) A perfectly matched layer absorbing boundary condition for the second-order seismic wave equation. Geophys J Int 154:146-153

Dumbser M, Kaser M, Toro E (2007) An arbitrary high order discontinuous Galerkin method for elastic waves on unstructured meshes V: local time stepping and p-adaptivity. Geophys J Int 171:695-717

Eriksson K, Johnson C (1991) Adaptive finite element methods for parabolic problems I: a linear model problem. SIAM J Numer Anal 28:43-77

Fei T, Larner K (1995) Elimination of numerical dispersion in finite difference modeling and migration by flux-corrected transport. Geophysics 60:1830-1842 
Frank DH, Jhon BS, Shira LB (1996) Application of the perfectly matched layer (PML) absorbing boundary condition to elastic wave propagation. J Acoust Soc Am 100(5):3061-3069

Igel H, Mora P, Riollet B (1995) Anisotropic wave propagation through finite-difference grids. Geophysics 60:1203-1216

Iserles A, Munthe-Kaas H, Norsett SP, Zanna A (2000) Lie group methods. Acta Numer 9:215-365

Kelly KR, Wave RW, Tretel S (1976) Synthetic seismograms: a finite-difference approach. Geophysics 41:2-27

Komatitsch D, Vilotte JP (1998) The spectral element method: an efficient tool to simulate the seismic responses of 2-D and 3-D geological structures. Bull Seismol Soc Am 88:368-392

Komatitsch D, Barnes C, Tromp J (2000) Simulation of anisotropic wave propagation based upon a spectral-element method. Geophysics 65:1251-1260

Kosloff D, Baysal E (1982) Forward modeling by a Fourier method. Geophysics 47:1402-1412

Krogstad S (2005) Generalized integrating factor methods for stiff PDEs. J Comput Phys 203:72-88

Li J, Tang G, Hu T (2010) Optimization of a precise integration method for seismic modeling based on graphic processing unit. Earthq Sci 23:387-393

Ma X, Yang DH, Liu FQ (2011) A nearly-analytic symplectically partitioned Runge-Kutta method for 2-D seismic wave equations. Geophys J Int 187:480-496

Minchev BV (2003) Exponential time differencing and Lie-group methods for stiff problems. In: The international conference on scientific computation and differential equations, Trondheim, June 30-July 4, 2003

Moszo P, Kristek J, Halada L (2000) 3-D fourth-order staggered-grid finite-difference schemes: stability and grid dispersion. Bull Seismol Soc Am 90(3):587-603

Munthe-Kaas H (1999) High order Runge-Kutta methods on manifolds. Appl Numer Math 29:115-227

Munthe-Kaas H, Zanna A (1997) Numerical integration of differential equations on homogeneous manifolds. http://hans.munthe-kaas.no/
work/Blog/Entries/1997/1/1_Article_Numerical_integration_of_ differential_equations_on_homogeneous_manifolds_files/munthekaas97nio.pdf. Accessed 15 May, 2013

Sun JQ, Qin MZ, Dai GD (2008) Exponential time difference method to solve the diffusion equation. J Numer Methods Comput Appl 29(4):261-266

Tang G, Hu T, Yang J (2007) Applications of a precise integration method in forward seismic modeling. In: The 77th SEG annual international meeting. Expanded abstract, 2130-2134

Tong P, Yang DH, Hua BL (2011) High accuracy wave simulationrevised derivation, numerical analysis and testing of a nearly analytic integration discrete method for solving acoustic equation. Int J Solids Struct 48:56-70

Versteeg RJ, Grau G (1991) The Marmousi experience. In: Proceedings of the EAGE workshop on practical aspects of seismic data inversion (Copenhagen, 1990), European Association of Exploration Geophysicists, Zeist

Yang DH, Teng JW, Zhang ZJ et al (2003) A nearly-analytic discrete method for acoustic and elastic wave equation. Bull Seismol Soc Am 93(2):882-890

Yang DH, Lu M, Wu RS, Peng JM (2004) An optimal nearly-analytic discrete method for 2-D acoustic and elastic wave equations. Bull Seismol Soc Am 94:1982-1991

Yang DH, Peng JM, Lu M, Tamas T (2006) Optimal nearly analytic discrete approximation to the scalar wave equation. Bull Seismol Soc Am 96:1114-1130

Yang DH, Song GJ, Chen S et al (2007) An improved nearly analytical discrete method: an efficient tool to simulate the seismic response of $2 \mathrm{D}$ porous structures. J Geophys Eng $4: 40-52$

Zhou H, Chen XF (2008) The localized boundary integral equation discrete wave-number method for simulating P-SV wave scattering by an irregular topography. Bull Seismol Soc Am 98:265-279 